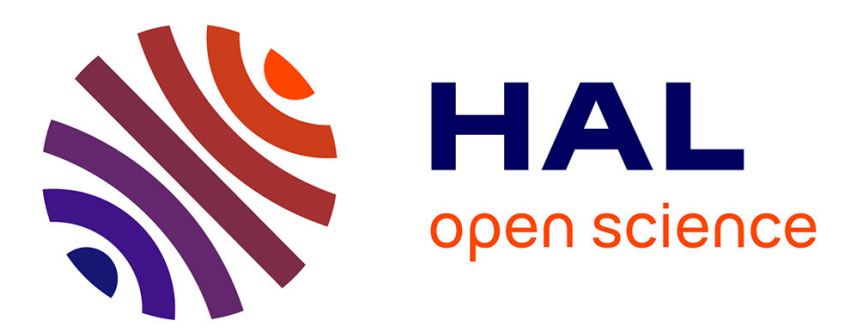

\title{
New Results on the Vibrating String with a Continuous Obstacle
}

\author{
Alain Bamberger, Michelle Schatzman
}

\section{To cite this version:}

Alain Bamberger, Michelle Schatzman. New Results on the Vibrating String with a Continuous Obstacle. SIAM Journal on Mathematical Analysis, 1983, 14 (3), pp.560-595. 10.1137/0514046 . hal-01570508

\section{HAL Id: hal-01570508 \\ https://hal.science/hal-01570508}

Submitted on $30 \mathrm{Jul} 2017$

HAL is a multi-disciplinary open access archive for the deposit and dissemination of scientific research documents, whether they are published or not. The documents may come from teaching and research institutions in France or abroad, or from public or private research centers.
L'archive ouverte pluridisciplinaire HAL, est destinée au dépôt et à la diffusion de documents scientifiques de niveau recherche, publiés ou non, émanant des établissements d'enseignement et de recherche français ou étrangers, des laboratoires publics ou privés. 


\title{
NEW RESULTS ON THE VIBRATING STRING WITH A CONTINUOUS OBSTACLE
}

\author{
A. BAMBERGER ${ }^{\dagger}$ AND M. SCHATZMAN ${ }^{\dagger \neq}$
}

Abstract. We give an explicit formula which describes the solution of the problem of the linear elastic string vibrating against a plane obstacle without loss of energy. This formula allows us to prove continuous dependence on the initial data; a regularity result in some bounded variation spaces is given. A numerical scheme is deduced from the explicit formula.

Finally we prove the weak convergence of a subsequence of solutions of the penalized problem to a "weak" solution (i.e. one which does not necessarily conserve energy) of the problem with an obstacle when the obstacle is arbitrary; when the obstacle is plane, all the sequence strongly converges to the solution of the obstacle problem which conserves the energy.

\section{Introduction.}

1.1. Presentation of the problem and the results. This paper aims to give some new results on vibrating strings with obstacles. The model is the same as in [5], but as it appears necessary to elucidate several points of the modelization which was exposed there, we shall give it from the beginning.

We consider the small transverse vibrations of a string that is constrained to be on one side of a material obstacle. Let the transverse displacement at time $t$ of the material point of the string with coordinate $x$ be denoted by $u(x, t)$. If the string were free, i.e., if there was no obstacle, then $u$ would satisfy the wave equation

$$
\square u \equiv u_{t t}-u_{x x}=0 \text {. }
$$

We assume that the obstacle has position $\varphi(x)$. We translate the requirement that the string stay on one side of the obstacle into the inequality

$$
u(x, t) \geq \varphi(x) \quad \forall x, t .
$$

When the string does not touch the obstacle, its motion satisfies the wave equation, and thus

$$
\operatorname{supp} \square u \subset\{(x, t): u(x, t)=\varphi(x)\} .
$$

We require that the string does not stick to the obstacle; this can be translated as

$$
\square u \geq 0 \text {, }
$$

which means that the obstacle does not exert a downward force on the string.

Notice that (3) is essentially equivalent to subsonic propagation of interactions. To see this, let $t=\sigma(x)$ be a curve which separates a region $\Re$ on the half-plane $\mathbb{R} \times(0, \infty)$ in two open regions $\Re^{+}$and $\Re^{-}$where $\square u$ vanishes. Suppose that $u^{+}=\left.u\right|_{\mathscr{R}^{+}}$and $u^{-}=\left.u\right|_{\mathscr{R}^{-}}$are sufficiently smooth, and that

$$
\begin{gathered}
u^{ \pm}(x, \sigma(x))=\varphi(x), \\
u^{ \pm}(x, t) \geq \varphi(x) \quad \forall(x, t) \in \Re .
\end{gathered}
$$

${ }^{\ddagger}$ Centre de Mathématiques Appliquées, Ecole Polytechnique, Route de Saclay, 91128 Palaiseau Cedex, France.

₹Part of this paper was written when this author was visiting the Universities of Michigan (Ann Arbor), Wisconsin (Madison) and California (Berkeley). The research of this author was sponsored by the U. S. Army under contract DAAG29-75-C-0024, and by the U. S. Air Force Office of Scientific Research under contract C-F49620-79-C-0128. 
Then we can compute $\square u$ in the sense of distributions, with $\psi$ a test function:

$\langle\square u, \psi\rangle=-\left\langle\frac{\partial u}{\partial t}, \frac{\partial \psi}{\partial t}\right\rangle+\left\langle\frac{\partial u}{\partial x}, \frac{\partial \psi}{\partial x}\right\rangle$

$$
=\int\left[\left(\frac{\partial u^{+}}{\partial t}-\frac{\partial u^{-}}{\partial t}\right)(x, \sigma(x))+\left(\frac{\partial u^{+}}{\partial x}-\frac{\partial u^{-}}{\partial x}\right)(x, \sigma(x)) \sigma^{\prime}(x)\right] \psi(x, \sigma(x)) d x .
$$

Relation (4) can be differentiated with respect to $x$, and implies

$$
\left(\frac{\partial u^{+}}{\partial x}-\frac{\partial u^{-}}{\partial x}\right)(x, \sigma(x))=-\sigma^{\prime}(x)\left(\frac{\partial u^{+}}{\partial t}-\frac{\partial u^{-}}{\partial t}\right)(x, \sigma(x)) .
$$

Introducing (7) into (8), we get

$$
\langle\square u, \psi\rangle=\int\left(\frac{\partial u^{+}}{\partial t}-\frac{\partial u^{-}}{\partial t}\right)(x, \sigma(x))\left(1-\sigma^{\prime 2}(x)\right) \psi(x, \sigma(x)) d x .
$$

But hypotheses (4) and (5) ensure that

$$
\frac{\partial u^{+}}{\partial t}(x, \sigma(x)) \geq 0 \quad \text { and } \quad \frac{\partial u^{-}}{\partial t}(x, \sigma(x)) \leq 0 .
$$

Therefore, $\square u$ is nonnegative if and only if $\left|\sigma^{\prime}\right|$ is almost everywhere smaller than 1 .

It is not enough to suppose that conditions (1), (2) and (3) are satisfied, as nothing has been said of the evolution of the energy of the string during the collision with the obstacle.

The hypothesis that will be made is that the energy is conserved. This requirement should be analysed from a mathematical point of view as follows: The condition must be local, because the propagation properties of hyperbolic equations suggest it, and it must be satisfied wherever in the $x, t$ half-plane the free wave equation is satisfied. Thus, multiplying by $\partial u / \partial t$ the relation

$$
\square u=0 \quad \text { on } \Re,
$$

where $R$ is an open region such that (8) is satisfied, we obtain a relation in divergence form:

$$
\frac{\partial}{\partial t}\left(\left|\frac{\partial u}{\partial t}\right|^{2}+\left|\frac{\partial u}{\partial x}\right|^{2}\right)-\frac{\partial}{\partial x}\left(2 \frac{\partial u}{\partial t} \frac{\partial u}{\partial x}\right)=0 \quad \text { in } \Re .
$$

The operations by which we deduce (9) out of (8) are valid if $\partial u / \partial t$ and $\partial u / \partial x$ are locally square-integrable in $\mathbb{R} \times(0, \infty)$.

The energy condition we shall impose is

$$
\frac{\partial}{\partial t}\left(\left|\frac{\partial u}{\partial t}\right|^{2}+\left|\frac{\partial u}{\partial x}\right|^{2}\right)-\frac{\partial}{\partial x}\left(2 \frac{\partial u}{\partial t} \frac{\partial u}{\partial x}\right)=0
$$

$$
\text { in the sense of distribution on } \mathbb{R} \times(0, \infty) \text {. }
$$

We could alternatively write it as

$$
S_{u} \stackrel{\text { def }}{=}\left(-2 u_{x} u_{t}, u_{x}^{2}+u_{t}^{2}\right), \quad \nabla \cdot S_{u}=0 .
$$

Here, the first component of the vector field $S_{u}$ is the energy density flux, and the second component of the vector field $S_{u}$ is the energy density.

Notice that (10) cannot be deduced by multiplying (3) by $\partial u / \partial t$, as $\partial u / \partial t$ must be expected to be discontinuous on the support of $\square u$. 
For initial conditions such that the free solution corresponding to them is locally of bounded energy, it was proved in [5] that the Cauchy problem (1)-(3) and (11) possesses a unique solution if the function $\varphi$ is convex.

The approach which led to condition (11) is essentially a mathematical one; from the mechanical point of view, one would like to know if (11) implies that the velocity of the string after collision is the opposite of the velocity of the string before collision. The answer is affirmative, but one has to give a meaning to

$$
\frac{\partial u}{\partial t}(x, t+0)=-\frac{\partial u}{\partial t}(x, t-0) \quad \text { if }(x, t) \in \operatorname{supp} \square u \text {. }
$$

This was the purpose of $[1$, part V], where it was shown that if

$$
\sigma \text { is Lipschitz continuous on } \mathbb{R} \text {, with Lipschitz }
$$$$
\text { constant } 1 \text {, and } \sigma \geq 0 \text { on } \mathbb{R} \text {, }
$$

and if (3) is satisfied, then right and left derivatives can be defined almost everywhere on the noncharacteristic parts of the curve $t=\sigma(x)$.

Moreover, if (11) holds, then for all $\sigma$ satisfying (13), we have:

$$
\left|\frac{\partial^{+} u}{\partial t}(x, \sigma(x))\right|=\left|\frac{\partial^{-} u}{\partial t}(x, \sigma(x))\right| \text { a.e. on }\left\{x:\left|\sigma^{\prime}(x)\right|<1\right\} \text {. }
$$

We shall prove in $\$ 2$ the following explicit formula in the case of the plane obstacle.

Let $w$ be the free solution of the wave equation

$$
\begin{aligned}
& \square w=0, \\
& w(x, 0)=u_{0}(x), \\
& w_{t}(x, 0)=u_{1}(x) .
\end{aligned}
$$

Let the obstacle be $\varphi=0$, and let the backward wave cone be

$$
T_{x, t}^{-} \stackrel{\text { def }}{=}\left\{\left(x^{\prime}, t^{\prime}\right): 0 \leq t^{\prime} \leq t-\left|x-x^{\prime}\right|\right\} .
$$

Let us denote by $r^{-}$the negative part of a number $r^{-}=\sup (-r, 0)$. Then the solution of the problem (1)-(3) and (11) is given by

$$
u(x, t)=w(x, t)+2 \sup \left\{\left(w\left(x^{\prime}, t^{\prime}\right)\right)^{-}:\left(x^{\prime}, t^{\prime}\right) \in T_{x, t}^{-}\right\} .
$$

This formula shortens considerably a previous proof [2] of continuous dependence on data, and is the key for the numerical scheme studied in $\$ 3$. We shall give in $\$ 4$ a regularity theorem in spaces of bounded variation, in the case of a general concave obstacle.

In $\S 5$, we shall consider the functions $u_{\lambda}$ which solve the problem

$$
\begin{aligned}
& \square u_{\lambda}-\frac{1}{\lambda}\left(u_{\lambda}-\varphi\right)^{-}=0, \\
& u_{\lambda}(x, 0)=u_{0}(x), \\
& \frac{\partial u_{\lambda}}{\partial t}(x, 0)=u_{1}(x) .
\end{aligned}
$$

In the first half of this section, we shall prove a weak convergence result, which does not depend on the shape of $\varphi$ nor on the regularity of the initial data. The limit function will satisfy a set of energy inequalities instead of (11). 
In the second half, we shall assume that the obstacle is plane, and that $d u_{0} / d x$ and $u_{1}$ are locally of bounded variation. Then the solution of (16) converges strongly in $H_{\text {loc }}^{1}\left(\mathbb{R} \times \mathbb{R}^{+}\right)$, and its limit is the unique solution of (1)-(3) and (11).

1.2. Notation and summary of previous results. We shall use throughout this paper the following notation and definitions:

$V$ is the set of functions $u$ such that

$$
\int_{-a}^{a}\left(\left|u_{x}(x, t)\right|^{2}+\left|u_{t}(x, t)\right|^{2}\right) d x \leq C(a, b)<+\infty \quad \forall a, b, \quad \forall t<b .
$$

$w$ is the free solution of the wave equation:

$$
\begin{aligned}
& \square w=0, \\
& w(x, 0)=u_{0}(x), \\
& w_{t}(x, 0)=u_{1}(x) .
\end{aligned}
$$

If we denote the closure of a set $A$ by $\operatorname{cl} A$, we define a set $E$ by

$$
E=\operatorname{cl}\{(x, t): w(x, t)<\phi(x)\} .
$$

$I$ is the domain of influence defined by

$$
I=\cup\left\{T_{x, t}^{+}:(x, t) \in E\right\},
$$

where $T_{x, t}^{+}$is the forward wave cone $\left\{\left(x^{\prime}, t^{\prime}\right): t^{\prime} \geq t+\left|x-x^{\prime}\right|\right\}$, and the boundary of $I$, called the line of influence, is given by

$$
\partial I=\{(x, t): t=\tau(x)\},
$$

where $\tau$ is Lipschitz continuous with Lipschitz constant 1

(see [5, proposition II.3] for the proof of this claim). The backward wave cone $T_{x, t}^{-}$is $\left\{\left(x^{\prime}, t^{\prime}\right): 0 \leq t^{\prime} \leq t-\left|x-x^{\prime}\right|\right\}$.

The characteristic coordinates $\xi$ and $\eta$ are given by

$$
\xi=\frac{x+t}{\sqrt{2}}, \quad \eta=\frac{-x+t}{\sqrt{2}}
$$

with the notation $\tilde{z}(\xi, \eta)=z((\xi-\eta) / \sqrt{2},(\xi+\eta) / \sqrt{2})$ for all functions of two variables $x$ and $t$.

We shall call problem $\left(\mathrm{P}_{\infty}\right)$ the following problem:

Given $u_{0} \in H_{\text {loc }}^{1}(\mathbb{R}), u_{1} \in L_{\text {loc }}^{2}(\mathbb{R})$ satisfying the compatibility condition

$$
\begin{aligned}
& u_{0}(x) \geq \varphi(x), \\
& u_{1}(x) \geq 0 \text { a.e. on }\left\{x: u_{0}(x)=\varphi(x)\right\}
\end{aligned}
$$

find $u$ in $V$ such that

(a) $u \geq \varphi$,

(b) $\operatorname{supp} \square u \subset\{(x, t): u(x, t)=\varphi(x)\}$,

(c) $\square u \geq 0$;

$$
\frac{\partial}{\partial t}\left(u_{x}^{2}+u_{t}^{2}\right)+\frac{\partial}{\partial x}\left(-2 u_{x} u_{t}\right)=0
$$

in the sense of distributions in $\mathbb{R} \times \mathbb{R}^{+}$;

$$
\begin{aligned}
& u(x, 0)=u_{0}(x), \\
& \frac{\partial u}{\partial t}(x, 0)=u_{1}(x) .
\end{aligned}
$$


The precise statement of the results of existence and uniqueness in [5] is as follows:

THEOREM 0. Problem $\left(\mathrm{P}_{\infty}\right)$ possesses a unique solution $u$ if $\varphi^{\prime \prime}$ is nonnegative. Moreover, this solution $u$ is the unique solution of the linear problem

$u \in V$,

$\square u_{\mid\{(x, t): t \neq \tau(x)\}}=0$,

$$
\begin{aligned}
& \frac{\partial u}{\partial t}(x, \tau(x)+0)=-\frac{\partial u}{\partial t}(x, \tau(x)-0) \text { a.e.on }\left\{x: \tau(x)>0 \&\left|\tau^{\prime}(x)\right|<1\right\}, \\
& u(x, 0)=u_{0}(x), \\
& \frac{\partial u}{\partial t}(x, 0)=u_{1}(x) .
\end{aligned}
$$

If $\mu$ is the measure defined by

$$
\langle\mu, \psi\rangle=-2 \int w_{t}(x, \tau(x))\left(1-\tau^{\prime}(x)^{2}\right) \psi(x, \tau(x)) d x,
$$

then the solution of (19) is given by the sum of the free solution $w$ and of a convolution

$$
u=w+\varepsilon * \mu
$$

where $\mathscr{E}$ is the elementary solution of the wave equation with support in the positive light cone:

$$
\mathcal{E}= \begin{cases}\frac{1}{2} & \text { on }\{(x, t): t \geq|x|\} \\ 0 & \text { elsewhere. }\end{cases}
$$

It will be useful to consider the problems $\left(\mathrm{P}_{x, t}\right)$, which are just $\left(\mathrm{P}_{\infty}\right)$ restricted to the backward wave cone $T_{x, t}^{-}$, with initial data given on $[x-t, x+t]$. Clearly, $u$ is a solution of $\left(\mathrm{P}_{\infty}\right)$ if and only if it is a solution of $\left(\mathrm{P}_{x, t}\right)$ for all $x \in \mathbb{R}, t>0$. The first result on the convergence of the penalty method for the string with an obstacle was proved by A. Bamberger [3].

An explicit formula for the string with a point obstacle was obtained by L. Amerio in [1] and by M. Schatzman in [6], with a different argument.

Continuous dependence on the data and convergence of the penalty method for the point obstacle are proved in [6]. See also the results of C. Citrini [4], where regularity assumptions are relaxed.

\section{The explicit formula. Continuous dependence on the initial data.}

2.1. The explicit formula for the infinite string. In the case of the zero obstacle (and more generally, the plane obstacle), the solution of $\left(\mathrm{P}_{\infty}\right)$ can be expressed by an explicit formula. We denote by $r^{-}=\sup (r, 0)$ the negative part of a number.

THEOREM 1. The unique solution of $\left(\mathrm{P}_{\infty}\right)$ when $\varphi=0$ is given by

$$
u(x, t)=w(x, t)+2 \sup _{\left(x^{\prime}, t^{\prime}\right) \in T_{x, t}^{-}}\left[w\left(x^{\prime}, t^{\prime}\right)\right]^{-} .
$$

Remark 2. If the obstacle is plane, i.e., if $\varphi(\mathrm{x})=\alpha \mathrm{x}+\beta$, then (28) can be generalized to

$$
u(x, t)=w(x, t)+2 \sup _{\left(x^{\prime}, t^{\prime}\right) \in T_{x, t}^{-}}\left[w\left(x^{\prime} t^{\prime}\right)-\varphi\left(x^{\prime}\right)\right]^{-} .
$$

To deduce (29) from (28) it is enough to consider $u-\varphi$, and notice that $\square \varphi=0$. 
The proof of Theorem 1 comes in several steps. The first step is the following result:

LEMma 3. The set where $\sup \left\{\left[w\left(x^{\prime}, t^{\prime}\right)\right]^{-}:\left(x^{\prime}, t^{\prime}\right) \in T_{x, t}^{-}\right\}$does not vanish is the interior of the domain of influence $I$.

Proof. If $w\left(x^{\prime}, t^{\prime}\right)<0$ for some $\left(x^{\prime}, t^{\prime}\right)$ in the backward cone $T_{x, t}^{-}$, then $(x, t)$ belongs to the forward cone $T_{x^{\prime}, t^{\prime}}^{+}$, the vertex of which is in the interior of $E$. Thus $(x, t)$ is in the interior of $I$. Conversely, if $(x, t)$ belongs to the interior of $I$, then there exists a point $\left(x^{\prime}, t^{\prime}\right)$ in the interior of $E$ such that $(x, t)$ belongs to the interior of $T_{x^{\prime}, t^{\prime}}^{+}$. We can choose this $\left(x^{\prime}, t^{\prime}\right)$ such that $w\left(x^{\prime}, t^{\prime}\right)$ is strictly negative, because the set of $\left(x^{\prime}, t^{\prime}\right)$ such that $w\left(x^{\prime}, t^{\prime}\right)<0$ is dense in the interior of $E$. Therefore, $\sup _{\left(x^{\prime}, t^{\prime}\right) \in T_{x, t}^{-}}\left[w\left(x^{\prime}, t^{\prime}\right)\right]^{-}>0$.

Let us define

$$
k(x, t)=\inf \left\{w\left(x^{\prime}, t^{\prime}\right):\left(x^{\prime}, t^{\prime}\right) \in T_{x, t}^{-}\right\} .
$$

Then, thanks to Lemma 3, we have, if $u$ is defined by (28),

$$
u(x, t)=\left\{\begin{array}{l}
w(x, t) \quad \text { for } t \leq \tau(x), \\
w(x, t)-2 k(x, t) \text { for } t \geq \tau(x) .
\end{array}\right.
$$

LEMMA 4. Let $u_{0}$ and $u_{1}$ satisfy the compatibility conditions (22), and let I be nonempty. Then the function $k$ satisfies

$$
\square k=0 \quad \text { in the interior of } I .
$$

Proof. Let us extend $w$ to the whole plane $\mathbb{R} \times \mathbb{R}$, by solving the (backward) wave equation

$$
\begin{aligned}
& w(x, 0)=u_{0}(x), \\
& w_{t}(x, 0)=u_{1}(x), \\
& \square w=0 \text { for } t<0, x \in \mathbb{R} .
\end{aligned}
$$

The assumption that $I$ is not empty implies that, on the line of influence,

$$
\begin{array}{ll}
w(x, \tau(x))=0 & \text { if }\left|\tau^{\prime}(x)\right|<1, \\
w_{t}(x, \tau(x))<0 & \text { a.e. on }\left\{x:\left|\tau^{\prime}(x)\right|<1\right\} .
\end{array}
$$

We shall prove that $w(x, t) \geq 0$ for $t \leq \tau(x)$, by essentially the same argument as in [5, Thm. IV.2]. For the convenience of the reader, let us sketch it here.

Let $\left.U=\{x: w(x, \tau(x))>\varphi(x)\}=\cup_{i}\right] a_{i}, b_{i}$, where the open sets $] a_{i}, b_{i}[$ are the connected components of $U$. Then [5, Lemma II.6] tells us that

$$
\tau(x)=\min \left(\tau\left(a_{i}\right)+x-a_{i}, \tau\left(b_{i}\right)+b_{i}-x\right) \quad \forall x \in\left[a_{i}, b_{i}\right] .
$$

Therefore if we set

$$
\begin{array}{ll}
\xi_{i}=\frac{a_{i}+\tau\left(a_{i}\right)}{\sqrt{2}}, & \xi_{i}^{\prime}=\frac{b_{i}+\tau\left(b_{i}\right)}{\sqrt{2}}, \\
\eta_{i}=\frac{-a_{i}+\tau\left(a_{i}\right)}{\sqrt{2}}, & \eta_{i}^{\prime}=\frac{-b_{i}+\tau\left(b_{i}\right)}{\sqrt{2}}
\end{array}
$$

the line of influence in characteristic coordinates is such that

$$
Y(\xi)= \begin{cases}\eta_{i} & \text { if } \xi \in\left[\xi_{i}, \xi_{i}^{\prime}\right), \\ {\left[\eta_{i}^{\prime}, \eta_{i}\right]} & \text { if } \xi=\xi_{i}^{\prime},\end{cases}
$$


if $Y$ is the multivalued mapping (see Fig. 1) defined by

$$
\eta \in Y(\xi) \Leftrightarrow \frac{\xi+\eta}{\sqrt{2}}=\sigma\left(\frac{\xi-\eta}{\sqrt{2}}\right) .
$$

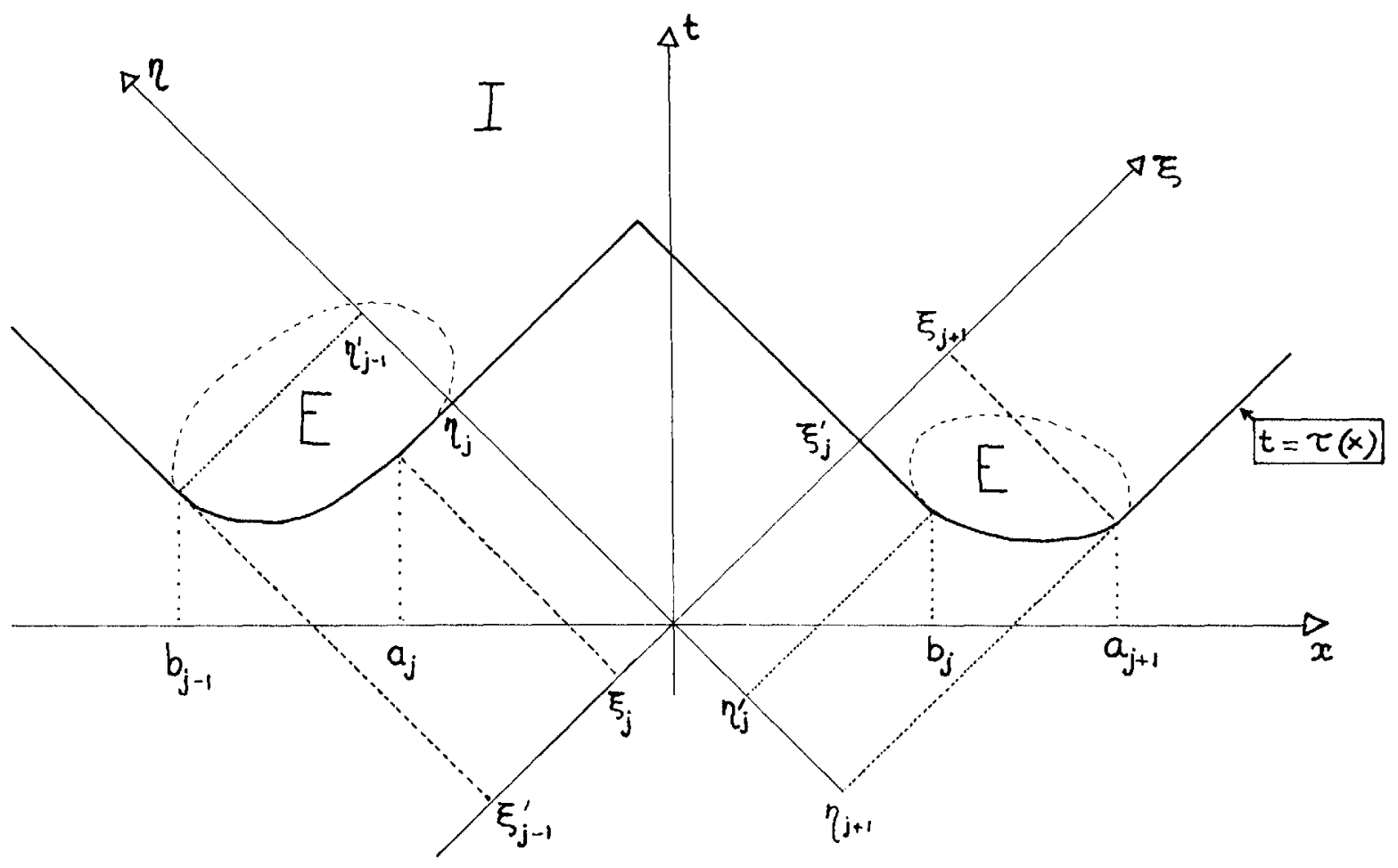

FIG. 1.The sets $E$ and $I$, the influence line $t=\tau(x)$, the intervals $\left(a_{j}, b_{j}\right)$, and the characteristic coordinates, with the $\xi_{j}, \xi_{j}^{\prime}, \eta_{j}, \eta_{j}^{\prime}$.

Let $\tilde{w}(\xi, \eta)=f(\xi)+g(\eta)$, where $f$ and $g$ are in $H_{\text {loc }}^{1}(\mathbb{R})$. From (35), we deduce $f(\xi)+$ $g\left(\eta_{i}^{\prime}\right) \geq 0$ for $\xi_{i} \leq \xi \leq \xi_{i}^{\prime}$ and from (34), $f(\xi)+g\left(\eta_{i}\right) \geq 0$ for $\xi_{i} \leq \xi \leq \xi_{i}^{\prime}$. As we must have $f\left(\xi_{i}\right)+g\left(\eta_{i}\right)=0=f\left(\xi_{i}^{\prime}\right)+g\left(\eta_{i}^{\prime}\right)=0$, by definition of $U, \xi_{i}, \xi_{i}^{\prime}, \eta_{i}$ and $\eta_{i}^{\prime}$, then

$$
f\left(\xi_{i}\right)=f\left(\xi_{i}^{\prime}\right) \leq f(\xi) \quad \forall \xi \in\left[\xi_{i}, \xi_{i}^{\prime}\right]
$$

Similarly,

$$
g\left(\eta_{i}\right)=g\left(\eta_{i}^{\prime}\right) \leq g(\eta) \quad \forall \eta \in\left[\eta_{i}^{\prime}, \eta_{i}\right] .
$$

On $C$, the complement of the set $\cup_{i}\left[\xi_{i}, \xi_{i}^{\prime}\right]$, we have

$$
f^{\prime}(\xi) \leq 0 \text { a.e. }
$$

The simplest way to see this is to notice that $Y$ is one-valued on $C$, and that

$$
\begin{array}{ll}
f(\xi)+g(Y(\xi))=0 & \text { on } C, \\
f\left(\xi^{\prime}\right)+g(Y(\xi)) \geq 0 & \text { for } \xi^{\prime} \leq \xi .
\end{array}
$$

Let us now evaluate $f(\xi)+g(\eta)$. Suppose first that $X(\eta)=Y^{-1}(\eta)$ is one-valued. Then

$$
\begin{aligned}
f(\xi)+g(\eta)=g(\eta) & +f(X(\eta))-\int_{\xi}^{X(\eta)} 1_{C} f^{\prime}\left(\xi^{\prime}\right) d \xi^{\prime} \\
& -\sum_{i}\left[f\left(\min \left(\xi_{i}^{\prime}, X(\eta)\right)\right)-f\left(\max \left(\xi_{i}, \xi\right)\right)\right],
\end{aligned}
$$


where the summation is extended to the indices such that $\left[\xi_{i}, \xi_{i}^{\prime}\right]$ intersects $[\xi, X(\eta)]$. We have:

$$
\begin{aligned}
& g(\eta)+f(X(\eta)) \geq 0 \\
& \int_{\xi}^{X(\eta)} 1_{C} f^{\prime}\left(\xi^{\prime}\right) d \xi^{\prime} \leq 0 \quad \text { by }(39) .
\end{aligned}
$$

As $X(\eta)$ is one-valued, it is not contained in the interior of an interval $\left[\xi_{i}, \xi_{i}^{\prime}\right]$. Thus

$$
\min \left[\xi_{i}^{\prime}, X(\eta)\right)=\xi_{i}^{\prime} \quad \text { if }\left[\xi_{i}, \xi_{i}^{\prime}\right] \cap[\xi, X(\eta)] \neq \varnothing
$$

and, if $\xi \notin\left[\xi_{i}, \xi_{i}^{\prime}\right]$, the corresponding term in the sum vanishes. For $\xi$ in $\left[\xi_{i}, \xi_{i}^{\prime}\right]$, the term in the sum is $f\left(\xi_{i}^{\prime}\right)-f(\xi)$, which is not positive, by (37). Therefore, the expression (40) is nonnegative for $\xi \leq X(\eta)$. If we suppose that $X(\eta)=\left[\xi_{j}, \xi_{j}^{\prime}\right]$, we have to study the expression

$$
g(\eta)+f\left(\xi_{j}^{\prime}\right)-\int_{\xi}^{\xi_{j}^{\prime}} 1_{C} f^{\prime}\left(\xi^{\prime}\right) d \xi^{\prime}-\sum_{i}\left[f\left(\min \left(\xi_{i}^{\prime}, \xi_{j}^{\prime}\right)\right)-f\left(\max \left(\xi_{i}, \xi\right)\right)\right]
$$

and the result still holds, i.e.,

$$
w(x, t) \geq 0 \text { for } t \leq \tau(x) .
$$

Thanks to (41), we may redefine $k$ as

$$
k(x, t)=\inf \left\{w\left(x^{\prime}, t^{\prime}\right): t^{\prime} \leq t-\left|x-x^{\prime}\right|\right\},
$$

or still, in characteristic coordinates,

$$
\tilde{k}(\xi, \eta)=\inf \left\{f\left(\xi^{\prime}\right)+g\left(\eta^{\prime}\right): \xi^{\prime} \leq \xi \& \eta^{\prime} \leq \eta\right\} .
$$

Then, it is immediate that

$$
\tilde{k}(\xi, \eta)=\inf \left\{f\left(\xi^{\prime}\right): \xi^{\prime} \leq \xi\right\}+\inf \left\{g\left(\eta^{\prime}\right): \eta^{\prime} \leq \eta\right\},
$$

which proves the claim of Lemma 4.

We shall now prove that $u$, defined by (28), satisfies the transmission condition (15) across the line of influence.

LEMMA 5. If $u$ is defined by (28), then almost everywhere on $\left\{x:\left|\tau^{\prime}(x)\right|<1\right\}$

$$
\frac{\partial u}{\partial t}(x, \tau(x)+0)=-\frac{\partial u}{\partial t}(x, \tau(x)-0) .
$$

Proof. Let $A=\left\{x:\left|\tau^{\prime}(x)\right|<1\right\}$. Then, almost everywhere on $A$, by [1, A.2],

$$
w_{x}(x, \tau(x)) \text { and } w_{t}(x, \tau(x)) \text { exist. }
$$

Let $x$ be a point satisfying (45), and let us denote

$$
w_{x}(x, \tau(x))=a, \quad w_{t}(x, \tau(x))=b, \quad \tau^{\prime}(x)=m .
$$

Then

$$
a+m b=0, \quad b \leq 0
$$

and

$$
w\left(x^{\prime}, t^{\prime}\right)=a\left(x^{\prime}-x\right)+b\left(t^{\prime}-\tau(x)\right)+\varepsilon\left(x^{\prime}-x, t^{\prime}-\tau(x)\right),
$$

where $\varepsilon$ satisfies

$$
\lim _{|r|+|s| \rightarrow 0} \frac{\varepsilon(r, s)}{|r|+|s|}=0
$$


We have

$$
\inf \left\{a\left(x^{\prime}-x\right)+b\left(t^{\prime}-\tau(x)\right):\left(x^{\prime}, t^{\prime}\right) \in T_{x, t}^{-}\right\}=b(t-\tau(x)),
$$

and therefore,

$$
\begin{aligned}
& b(t-\tau(x))-\sup \left\{\left|\varepsilon\left(x^{\prime}-x, t^{\prime}-t\right)\right|: \tau\left(x^{\prime}\right) \leq t^{\prime} \leq t-\left|x-x^{\prime}\right|\right\} \\
& \quad \leq k(x, t) \leq b(t-\tau(x))+|\varepsilon(0, t-\tau(x))|
\end{aligned}
$$

As $\left|\tau^{\prime}(x)\right|<1$, we have

$$
\lim _{t \downarrow \tau(x)}\left[\sup \left\{\left|\varepsilon\left(x^{\prime}-x, t^{\prime}-t\right)\right|: \tau\left(x^{\prime}\right) \leq t^{\prime} \leq t-\left|x-x^{\prime}\right|\right\} /(t-\tau(x))\right]=0,
$$

and we deduce from (46) that

$$
\lim _{t \downarrow \tau(x)} \frac{u(x, t)-u(x, \tau(x))}{t-\tau(x)}=-w_{t}(x, \tau(x))
$$

under the assumption (45).

Conclusion of the proof of Theorem 1. Lemmas 3,4 and 5 imply that the function $u$ defined by (28) solves the linear problem (24), up to the condition $u \in V$. Therefore it remains to check this last condition. If we take into account the formula (43), let us show that $k$ is in $V$.

We know that $f$ is in $H_{\text {loc }}^{1}(\mathbb{R})$; let

$$
\hat{f}(\xi)=\inf \left\{f\left(\xi^{\prime}\right): \xi^{\prime} \leq \xi\right\} .
$$

Then, we can compute the derivative of $\hat{f}(\xi)$ almost everywhere:

$$
\hat{f}^{\prime}(\xi)=\left\{\begin{array}{l}
0 \quad \text { if } f(\xi)>\hat{f}(\xi) \text { or if } f^{\prime}(\xi) \geq 0, \\
f^{\prime}(\xi) \text { if } f(\xi)=\hat{f}(\xi) \text { and if } f^{\prime}(\xi)<0 .
\end{array}\right.
$$

We deduce from $(47)$ that $\hat{f}$ is in $H_{\text {loc }}^{1}(\mathbb{R})$. Similarly, $\hat{g}$ is in $H_{\text {loc }}^{1}(\mathbb{R})$. The function $k$ which can be written as

$$
k(x, t)=\hat{f}\left(\frac{x+t}{\sqrt{2}}\right)+\hat{g}\left(\frac{-x+t}{\sqrt{2}}\right)
$$

will therefore be in $V$, i.e.,

$$
\int_{-a}^{a}\left(\left|k_{x}(x, t)\right|^{2}+\left|k_{t}(x, t)\right|^{2}\right) d x \leq C(a, b) \quad \forall a, b, \quad \forall t \geq 0
$$

and thus $u$ is in $V$.

\subsection{Continuous dependence on the data.}

Corollary 6. The map $\left(u_{0}, u_{1}\right) \rightarrow u$ which to an element of $H_{\mathrm{loc}}^{1}(\mathbb{R}) \times L_{\mathrm{loc}}^{2}(\mathbb{R})$ satisfying the compatibility condition (22) associates the solution of $\left(\mathrm{P}_{\infty}\right)$ is continuous from $H_{\mathrm{loc}}^{1}(\mathbb{R}) \times L_{\mathrm{loc}}^{2}(\mathbb{R})$ equipped with the strong topology to

$$
W_{\mathrm{loc}}^{1, p}\left([0,+\infty) ; L_{\mathrm{loc}}^{2}(\mathbb{R})\right) \cap L_{\mathrm{loc}}^{p}\left([0,+\infty) ; H_{\mathrm{loc}}^{1}(\mathbb{R})\right)
$$

equipped with the strong topology, for all finite $p$.

Proof. We have at once the continuity from $H_{\text {loc }}^{1}(\mathbb{R}) \times L_{\text {loc }}^{2}(\mathbb{R})$ to $C^{0}\left(\mathbb{R} \times \mathbb{R}^{+}\right)$. 
The topology on $W_{\mathrm{loc}}^{1, p}\left([0,+\infty) ; L_{\mathrm{loc}}^{2}(\mathbb{R})\right) \cap L_{\mathrm{loc}}^{p}\left([0,+\infty) ; H_{\mathrm{loc}}^{1}(\mathbb{R})\right)$ is defined by the seminorms for $A, B>0$

$$
q_{A B p}(u)=|u(0,0)|+\left(\int_{0}^{B}\left[\int_{-A}^{A}\left(u_{x}^{2}+u_{t}^{2}\right)(x, t) d x\right]^{p / 2}\right)^{1 / p} .
$$

The topology on $H_{\mathrm{loc}}^{1}(\mathbb{R}) \times L_{\text {loc }}^{2}(\mathbb{R})$ is defined by the seminorms for $A>0$

$$
p_{A}\left(u_{0}, u_{1}\right)=\left|u_{0}(0)\right|+\left(\int_{-A}^{A}\left(\left|\frac{d u_{0}}{d x}\right|^{2}+\left|u_{1}\right|^{2}\right) d x\right)^{1 / 2} \text {. }
$$

It has been proved in $[5, \S \mathrm{IV} .2]$ that for solutions of $\left(\mathrm{P}_{\infty}\right)$ with zero obstacle,

$$
\left|\frac{\partial u}{\partial \xi}(x, t)\right|=\left|\frac{\partial u}{\partial \xi}(x+t, 0)\right|, \quad\left|\frac{\partial u}{\partial \eta}(x, t)\right|=\left|\frac{\partial u}{\partial \eta}(x-t, 0)\right| .
$$

Therefore

$$
\begin{aligned}
\int_{-A}^{A}\left(\left|u_{x}\right|^{2}+\left|u_{t}\right|^{2}\right)(x, t) d x & =\int_{-A}^{A}\left(\left|u_{\xi}\right|^{2}+\left|u_{\eta}\right|^{2}\right)(x, t) d x \\
& \leq \int_{-A-t}^{A+t}\left(\left|\frac{d u_{0}}{d x}\right|^{2}+\left|u_{1}\right|^{2}\right) d x \quad \forall A, t>0 .
\end{aligned}
$$

Let $q_{A B \infty}$ be the seminorm

$$
q_{A B \infty}(v)=v(0,0)+\operatorname{esssup}_{t \in[0, B]}\left(\int_{-A}^{A}\left(\left|v_{x}\right|^{2}+\left|v_{t}\right|^{2}\right)(x, t) d x\right)^{1 / 2} .
$$

Then (49) implies

$$
q_{A B \infty}(u) \leq p_{A+B}\left(u_{0}+u_{1}\right) .
$$

If $\left(u_{0}^{n}, u_{1}^{n}\right)$ is a sequence of initial data satisfying the compatibility condition (22) and converging to $\left(u_{0}, u_{1}\right)$ in $H_{\mathrm{loc}}^{1}(\mathbb{R}) \times L_{\mathrm{loc}}^{2}(\mathbb{R})$, then, as a consequence of (50),

$$
u^{n} \rightarrow u \text { in } H_{\text {loc }}^{1}\left(\mathbb{R} \times \mathbb{R}^{+}\right) \text {weakly, }
$$

and moreover, (48) implies that

$$
\begin{aligned}
& \int_{-A}^{A}\left|\frac{\partial u^{n}}{\partial \xi}(x, t)\right|^{2} d x \rightarrow \int_{-A}^{A}\left|\frac{\partial u}{\partial \xi}(x, t)\right|^{2} d x \quad \forall t, A>0 \\
& \int_{-A}^{A}\left|\frac{\partial u^{n}}{\partial \eta}(x, t)\right|^{2} d x \rightarrow \int_{-A}^{A}\left|\frac{\partial u}{\partial \eta}(x, t)\right|^{2} d x \quad \forall t, A>0 .
\end{aligned}
$$

Gathering (51), (52) and (53), we obtain

$$
u^{n} \rightarrow u \text { in } H_{\text {loc }}^{1}\left(\mathbb{R} \times \mathbb{R}^{+}\right) \text {strongly. }
$$

Thanks to Fubini's theorem, one has from (54)

$$
\left(u_{x}^{n}(\cdot, t), u_{t}^{n}(\cdot, t)\right) \rightarrow\left(u_{x}(\cdot, t), u_{t}(\cdot, t)\right) \quad \text { in }\left(L_{\text {loc }}^{2}(\mathbb{R})\right)^{2} \text { strongly, for almost all } t \geq 0 .
$$

The relation (55) together with the estimate

$$
q_{A B \infty}\left(u^{n}\right) \leq \sup _{n} p_{A+B}\left(u_{0}^{n}+u_{1}^{n}\right)<+\infty
$$


imply that $u^{n}$ converges to $u$ in the space $W_{\mathrm{loc}}^{1, p}\left([0,+\infty) ; L_{\mathrm{loc}}^{2}(\mathbb{R})\right) \cap L_{\mathrm{loc}}^{p}([0,+\infty)$; $\left.H_{\text {loc }}^{1}(\mathbb{R})\right)$.

Remark 7. The mapping $\left(u_{0}, u_{1}\right) \rightarrow u$ is not continuous to $W_{\text {loc }}^{1, \infty}\left([0,+\infty) ; L_{\text {loc }}^{2}(\mathbb{R})\right)$ $\cap L_{\text {loc }}^{\infty}\left([0,+\infty) ; H_{\text {loc }}^{1}(\mathbb{R})\right)$ which is the space $V$ defined in (17).

Take for instance the sequence of initial data

$$
u_{0}^{n}=1, \quad u_{1}^{n}=\frac{n+1}{n} .
$$

As these do not depend on $x$, the solution of $\mathrm{P}_{\infty}$ is

$$
u^{n}(x, t)= \begin{cases}1-\frac{n+1}{n} t & \text { if } t<\frac{n}{n+1}, \\ \frac{n+1}{n} t-1 & \text { if } t \geq \frac{n}{n+1},\end{cases}
$$

with the limit

$$
u(x, t)= \begin{cases}1-t & \text { if } t<1 \\ t-1 & \text { if } t \geq 1\end{cases}
$$

Then we may calculate $q_{A B \infty}\left(u^{n}-u\right)$ :

$$
q_{A B \infty}\left(u^{n}-u\right)= \begin{cases}0 & \text { if } B<\frac{n}{n+1}, \\ 2 \sqrt{2 A} & \text { if } B>\frac{n}{n+1} .\end{cases}
$$

Thus if $B>1, q_{A B \infty}\left(u^{n}-u\right)$ does not tend to zero as $n$ tends to infinity.

2.3. Application of the explicit formula to the finite string with fixed ends. The explicit formula (29) will allow us to give a simple construction of the solution of $\left(\mathrm{P}_{f}\right)$ where $\left(\mathrm{P}_{f}\right)$ is the problem of the vibrating string with fixed ends, and obstacle $\varphi=-K$ $<0$. The only modification with respect to $\left(\mathrm{P}_{\infty}\right)$ we shall require is that $u$ be in the space $L^{\infty}\left(0, T ; H_{0}^{1}(0, L)\right) \cap W^{1, \infty}\left(0, T ; L^{2}(0, L)\right)$ for all $T>0$.

In fact, $u$ will be in the space

$$
L^{\infty}\left((0, \infty) ; H_{0}^{1}(0, L)\right) \cap W^{1, \infty}\left((0, \infty) ; L^{2}(0, L)\right)
$$

because we can integrate $(10)$ on any rectangle $[0, L] \times[0, T]$, and we get the energy equality for arbitrary times $T$ :

$$
\int_{0}^{L}\left(\left|u_{x}(x, T)\right|^{2}+\left|u_{t}(x, T)\right|^{2}\right) d x=\int_{0}^{L}\left(\left|\frac{d u_{0}}{d x}\right|^{2}+\left|u_{1}\right|^{2}\right) d x .
$$

Let us define

$$
e=\left(\int_{0}^{L}\left(\left|\frac{d u_{0}}{d x}\right|^{2}+\left|u_{1}\right|^{2}\right) d x\right)^{1 / 2}
$$

Then

$$
|u(x, t)-u(0, t)|=\left|\int_{0}^{x} u_{x}\left(x^{\prime}, t\right) d x^{\prime}\right| \leq e \sqrt{x},
$$

and similarly

$$
|u(x, t)-u(L, t)| \leq e \sqrt{L-x} .
$$


Let $\alpha=K^{2} / e^{2}$. Then

$$
\forall t \in[0, \infty), \quad \forall x \in[0, \alpha) \cup(L-\alpha, L], \quad u(x, t)>-K,
$$

and $\square u$ cannot be supported in the strips $([0, \alpha) \cup(L-\alpha, L]) \times[0, \infty)$ (cf. Fig. 2). Let us extend the initial conditions $u_{0}, u_{1}$ to the interval $[-\alpha, L+\alpha]$ by:

$$
\begin{array}{lll}
u_{i}(-x)=-u_{i}(x) & \text { if } x \in[-\alpha, 0], & i=0,1, \\
u_{i}(L+x)=-u_{i}(L-x) & \text { if } x \in[0, \alpha], & i=0,1 .
\end{array}
$$

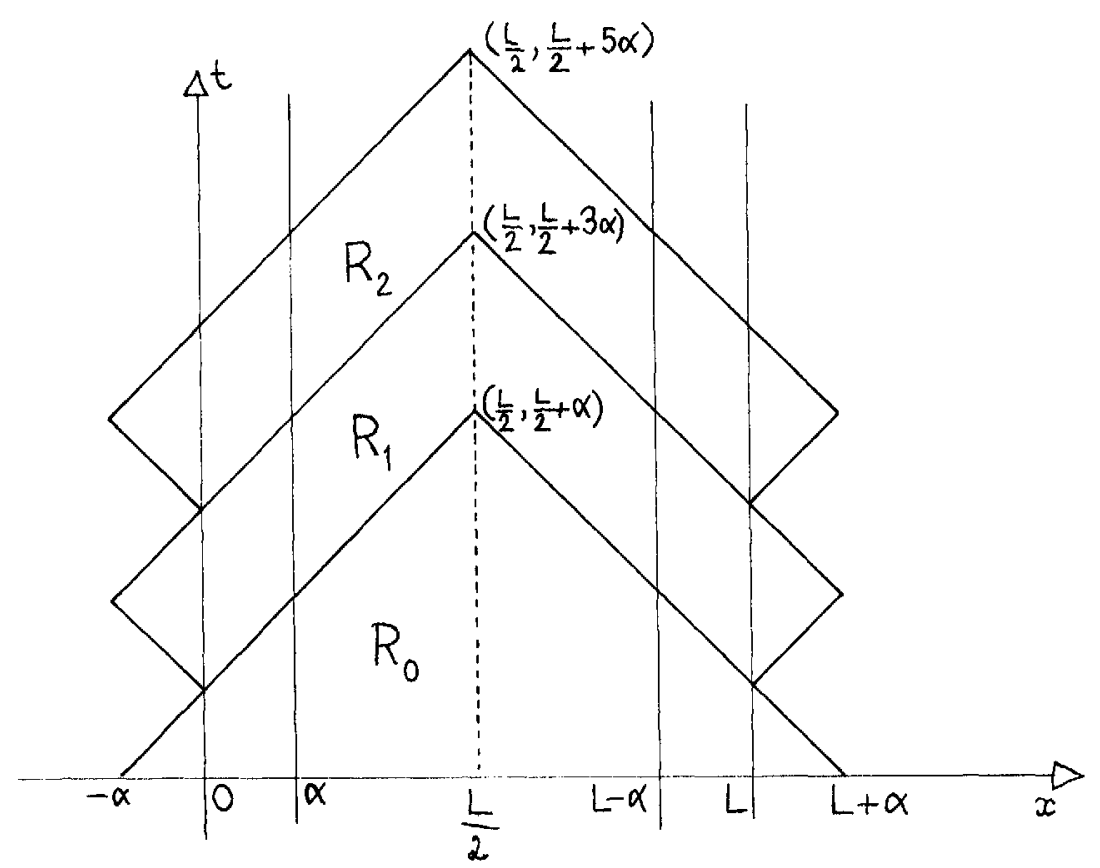

FIG. 2. The geometric construction used for the explicit formula in the case of the vibrating string with fixed ends, and a constant negative obstacle.

Then the corresponding free solution $w$ is defined on the cone $T_{L / 2, L / 2+\alpha}^{-}$, with the property that

$$
w(0, t)=0, \quad 0 \leq t \leq \alpha .
$$

Let $u$ be defined on $T_{L / 2, L / 2+\alpha}^{-}$by (29); then for $x=0, t \leq \alpha$,

$$
u(0, t)=w(0, t)+2 \sup _{T_{0, t}^{-}}\left\{\left[w\left(x^{\prime}, t^{\prime}\right)+K\right]^{-}\right\} .
$$

But, $w(0, t)=0$, and $T_{0, t}^{-}$is included in the strip $[-\alpha, \alpha] \times[0, \infty)$, so that $w>-K$ on this strip, and thus $u(0, t)=0$ on $[0, \alpha]$. Analogously, $u(L, t)=0$ on $[0, \alpha]$.

Therefore, (58) defines the solution of $\left(\mathrm{P}_{f}\right)$ on $T_{L / 2, L / 2+\alpha}^{-} \cap([0, L] \times[0, \infty))$.

Let us define by induction the solution of $\left(\mathrm{P}_{f}\right)$ on the region $R_{n}$ given by

$$
\begin{aligned}
R_{n}=\left\{(x, t) \in[0, L] \times[0, \infty): \frac{L}{2}+(2 n-1) \alpha-\left|x-\frac{L}{2}\right| \leq t\right. & \\
& \left.\leq \frac{L}{2}+(2 n+1) \alpha-\left|x-\frac{L}{2}\right|\right\} .
\end{aligned}
$$

We shall denote by $\sigma_{n}$ the function

$$
\begin{aligned}
& \sigma_{n}(x)=\frac{L}{2}+(2 n-1) \alpha-\left|x-\frac{L}{2}\right| \quad \text { if } x \in[0, L], \\
& \sigma_{n}(x)=\sigma_{n}(-x), \quad \sigma_{n}(L+x)=\sigma_{n}(L-x) \text { if } x \in[0, \alpha] .
\end{aligned}
$$


Suppose we know $u\left(x, \sigma_{n}(x)\right)$ for $x \in[0, L]$. Let

$$
\begin{array}{ll}
w_{n}\left(x, \sigma_{n}(x)\right)=u\left(x, \sigma_{n}(x)\right) & \text { if } x \in[0, L], \\
w_{n}\left(x, \sigma_{n}(x)\right)=-u\left(-x, \sigma_{n}(-x)\right) & \text { if } x \in[-\alpha, 0], \\
w_{n}\left(x, \sigma_{n}(x)\right)=-u\left(2 L-x, \sigma_{n}(2 L-x)\right) & \text { if } x \in[L ; L+\alpha], \\
\square w_{n}=0 . &
\end{array}
$$

The function $w_{n}$ is defined in the region

$$
\sigma_{n}(x) \leq t \leq(2 n+1) \alpha+\frac{L}{2}-\left|x-\frac{L}{2}\right|, \quad-\alpha \leq x \leq L+\alpha .
$$

Let us notice that the symmetry of the initial conditions in (60) implies

$$
w_{n}(0, t)=w_{n}(L, t)=0 \quad \text { for }(2 n-1) \alpha \leq t \leq(2 n+1) \alpha .
$$

Moreover, as $u\left(x, \sigma_{n}(x)\right) \geq-K, \forall x \in[0, L]$, and as $u$ satisfies the energy condition (10), we shall have

$$
\begin{array}{ll}
w_{n}(x, t) \geq-K & \text { for } \sigma_{n}(x) \leq t \leq(2 n+1) \alpha+-|x| \\
& \text { or for } \sigma_{n}(x) \leq t \leq(2 n+1) \alpha+-|L-x| .
\end{array}
$$

Let

$$
u(x, t)=w_{n}(x, t)+2 \sup \left[\left(w_{n}\left(x^{\prime}, t^{\prime}\right)+K\right)^{-}: \sigma_{n}\left(x^{\prime}\right) \leq t^{\prime} \leq t-\left|x-x^{\prime}\right|\right] .
$$

Thanks to (61) and (62), $u$ satisfies the boundary conditions. Therefore it solves the problem of the string with an obstacle on $R_{n}$, and the induction can be pursued.

3. A numerical scheme.

3.1. A numerical scheme in a backward cone for the zero obstacle. Let there be given initial data $u_{0}$ and $u_{1}$ on the interval $[-T, T]$. We seek an approximation to the problem $\left(\mathrm{P}_{0, T}\right)$ on the backward cone $T_{0, T}^{-}$.

Let $h=T / n$ be a step, and let us define discretized initial data $u_{0}^{n}$ and $u_{1}^{n}$ by the following formula, where $u_{0}^{h}$ is an affine interpolation, and $u_{1}^{h}$ is piecewise constant:

$$
\begin{array}{ll}
u_{0}^{h}(x)=\frac{1}{h}\left[u_{0}((p+1) h)-u_{0}(p h)\right](x-p h)+u_{0}(p h) & \text { if } x \in[p h,(p+1) h], \\
u_{1}^{h}(x)=\frac{1}{h} \int_{p h}^{(p+1) h} u_{1}\left(x^{\prime}\right) d x^{\prime} & \text { if } x \in[p h,(p+1) h] .
\end{array}
$$

The corresponding free solution $w^{h}$ is given by

$$
w^{h}(x, t)=\frac{1}{2}\left[u_{0}^{h}(x+t)+u_{0}^{h}(x-t)+\int_{x-t}^{x+t} u_{1}^{h}\left(x^{\prime}\right) d x^{\prime}\right] .
$$

Let us define

$$
\tilde{w}_{i, j}^{h}=w^{h}\left(\left(\frac{i-j}{2}\right) h,\left(\frac{i+j}{2}\right) h\right) \quad \text { for } 0 \leq \frac{i+j}{2} \leq n-\left|\frac{i-j}{2}\right| .
$$

Then $\tilde{w}_{i, j}^{h}$ satisfies the finite difference relation

$$
\tilde{w}_{i, j}^{h}=\tilde{w}_{i, j-1}^{h}+\tilde{w}_{i-1, j}^{h}-\tilde{w}_{i-1, j-1}^{h} .
$$


Let us define a function $\tilde{u}_{i, j}^{h}$ on our mesh by

$$
\tilde{u}_{i, j}^{h}=\tilde{w}_{i, j}^{h}+2 \max \left\{\left(\tilde{w}_{i^{\prime}, j^{\prime}}^{h}\right)^{-}: i^{\prime} \leq i, j^{\prime} \leq j, i^{\prime}+j^{\prime} \geq 0\right\} .
$$

We could define $\tilde{u}_{i, j}^{h}$ alternatively by

$$
\begin{aligned}
& \tilde{u}_{i, j}^{h}=\tilde{w}_{i, j}^{h}-2 \tilde{K}_{i, j}^{h}, \\
& \tilde{K}_{i, j}^{h}=\min \left(\tilde{K}_{i-1, j}^{h}, \tilde{K}_{i, j-1}^{h},-\left(\tilde{w}_{i, j}^{h}\right)^{-}\right), \\
& \tilde{K}_{i,-i}^{h}=0 \quad \text { if }-n \leq i \leq n .
\end{aligned}
$$

Notice that $\tilde{K}^{h}$ is not the discretization of $\tilde{k}$, the correction term in characteristic coordinates, but the discretization of $\tilde{k} .1 \tilde{I}$, where $\tilde{I}$ is the set $I$ in characteristic coordinates.

THEOREM 8. Let $u$ be the solution of $\left(\mathrm{P}_{0, T}\right)$ with zero obstacle, and let $\tilde{u}_{i, j}^{h}$ be defined by (67). Then:

$$
\max _{i, j}\left|\tilde{u}_{i, j}^{h}-u\left(\frac{i-j}{2} h, \frac{i+j}{2} h\right)\right| \leq C \sqrt{h},
$$

where $C$ depends only on the initial conditions.

Moreover, we have the following bounds on the (approximate) characteristic derivatives:

$$
\begin{aligned}
& \left|\tilde{u}_{i, j}^{h}-\tilde{u}_{i-1, j}^{h}\right| \leq \frac{1}{2}\left|\int_{(i-1) h}^{i h}\left(u_{0 x}+u_{1}\right)\left(x^{\prime}\right) d x^{\prime}\right|, \\
& \left|\tilde{u}_{i, j}^{h}-\tilde{u}_{i, j-1}^{h}\right| \leq \frac{1}{2}\left|\int_{-j h}^{(-j+1) h}\left(u_{0 x}-u_{1}\right)\left(x^{\prime}\right) d x^{\prime}\right| .
\end{aligned}
$$

Proof. Let us first evaluate $w_{i, j}^{h}-w\left(x^{\prime}, t^{\prime}\right)$ when $\left(x^{\prime}, t^{\prime}\right)$ is in the characteristic square centered on $((i-j) h / 2,(i+j) h / 2)$, with sides of length $h \sqrt{2}$, i.e.,

$$
\begin{aligned}
\frac{2 i-1}{2} h \leq x^{\prime}+t^{\prime} & \leq \frac{2 i+1}{2} h \text { and } \frac{-2 j-1}{2} h \leq x^{\prime}-t^{\prime} \leq \frac{-2 j+1}{2} h: \\
w\left(x^{\prime}, t^{\prime}\right)-\tilde{w}_{i, j}^{h} & =w\left(x^{\prime}, t^{\prime}\right)-w^{h}\left(\frac{i-j}{2} h, \frac{i+j}{2} h\right) \\
& =\frac{1}{2}\left[u_{0}\left(x^{\prime}+t^{\prime}\right)-u_{0}^{h}(i h)+u_{0}\left(x^{\prime}-t^{\prime}\right)-u_{0}^{h}(-j h)\right. \\
& \left.+\int_{x^{\prime}-t^{\prime}}^{x^{\prime}+t^{\prime}} u_{1}(y) d y-\int_{-j h}^{i h} u_{1}^{h}(y) d y\right],
\end{aligned}
$$

i.e.,

$$
\begin{aligned}
\left|w\left(x^{\prime}, t^{\prime}\right)-\tilde{w}_{i, j}^{h}\right| & \leq \frac{1}{2} \sqrt{\frac{h}{2}}\left[\left|\int_{i h}^{x^{\prime}+t^{\prime}}\left(u_{0 x}+u_{1}\right)^{2} d y\right|^{1 / 2}+\left|\int_{-j h}^{x^{\prime}-t^{\prime}}\left(u_{0 x}-u_{1}\right)^{2} d y\right|^{1 / 2}\right] \\
& \leq \frac{1}{2} \sqrt{\frac{h}{2}}\left[2 \int_{-T}^{T}\left[\left(u_{0 x}+u_{1}\right)^{2}+\left(u_{0 x}-u_{1}\right)^{2}\right] d y\right]^{1 / 2} \\
& =\sqrt{\frac{h}{2}}\left(\int_{-T}^{T}\left(u_{0 x}^{2}+u_{1}^{2}\right) d x\right)^{1 / 2} .
\end{aligned}
$$


We may then deduce from

$$
\left|w\left(x^{\prime}, t^{\prime}\right)-\tilde{w}_{i, j}^{h}\right| \leq \sqrt{\frac{h}{2}}\left(\int_{-T}^{T}\left(u_{0 x}^{2}+u_{1}^{2}\right) d x\right)^{1 / 2}
$$

that

$$
\begin{array}{r}
\left|\sup \left\{\left(\tilde{w}_{i, j}^{h}\right)^{-}: i^{\prime} \leq i, j^{\prime} \leq j, i+j \geq 0\right\}-\sup \left\{\left[w\left(x^{\prime}, t^{\prime}\right)\right]^{-}: 0 \leq t^{\prime}<t-\left|x-x^{\prime}\right|\right\}\right| \\
\leq \sqrt{\frac{h}{2}}\left(\int_{-T}^{T}\left(u_{0 x}^{2}+u_{1}^{2}\right) d x\right)^{1 / 2} .
\end{array}
$$

Let us note that $\tilde{w}_{i, j}^{h}=w((i-j) h / 2,(i+j) h / 2)$, because the approximation (64) is very particular.

This, in turn, gives

$$
\max _{i, j}\left|\tilde{u}_{i, j}^{h}-u\left(\frac{i-j}{2} h, \frac{i+j}{2} h\right)\right| \leq \sqrt{2 h}\left(\int_{-T}^{T}\left(u_{0 x}^{2}+u_{1}^{2}\right) d x\right)^{1 / 2}
$$

This completes the proof of (70).

We now turn to proving (71) and (72). Let us note first that if

$$
\tilde{k}_{i, j}^{h}=\min \left\{\tilde{w}_{i^{\prime}, j^{\prime}}^{h}: i^{\prime} \leq i, j^{\prime} \leq j, i^{\prime}+j^{\prime} \geq 0\right\},
$$

we can write $\tilde{k}_{i, j}^{h}$ alternatively as

$$
\tilde{k}_{i, j}^{h}=\min \left\{\tilde{w}_{i^{\prime}, j^{\prime}}^{h}: i^{\prime} \leq i, j^{\prime} \leq j\right\},
$$

because we know from (41) that $\tilde{w}_{i, j}^{h} \geq 0$ for $i+j \leq 0$, as long as we suppose that the domain of influence is not empty.

Relation (76) implies that

$$
\tilde{k}_{i, j}^{h}=\hat{f}^{h}(i)+\hat{g}^{h}(j)
$$

where

$$
\tilde{w}_{i, j}^{h}=f^{h}(i)+g^{h}(j) \text {, }
$$

and

$$
\hat{f}^{h}(i)=\min \left\{f^{h}\left(i^{\prime}\right): i^{\prime} \leq i\right\}, \quad \hat{g}^{h}(j)=\min \left\{g^{h}\left(j^{\prime}\right): j^{\prime} \leq j\right\} .
$$

Thus, (68) can be written as

$$
\tilde{u}_{i, j}^{h}=\tilde{w}_{i, j}^{h}+2\left[\hat{f}^{h}(i)+\hat{g}^{h}(j)\right]^{-}
$$

if $T$ is not empty. If $\hat{f}^{h}(i)+\hat{g}^{h}(j) \geq 0$, then $\hat{f}^{h}(i-1)+\hat{g}^{h}(j) \geq 0$, and (71) is immediate.

Suppose now that

$$
\hat{f}^{h}(i)+\hat{g}^{h}(j)<0 .
$$

We have two cases. In the first case,

$$
\hat{f}^{h}(i-1)+\hat{g}^{h}(j) \geq 0 .
$$

Then, necessarily

$$
f^{h}(i)=\hat{f}^{h}(i)<\hat{f}^{h}(i-1) \leq f^{h}(i-1)
$$


and thus,

$$
\begin{aligned}
\tilde{u}_{i, j}^{h}-\tilde{u}_{i-1, j}^{h} & =f^{h}(i)+g^{h}(j)-2 \hat{f}^{h}(i)-2 \hat{g}^{h}(j)-f^{h}(i-1)-g^{h}(j) \\
& =-\left[f^{h}(i)+\hat{g}^{h}(j)+\hat{f}^{h}(i-1)+\hat{g}^{h}(j)\right] .
\end{aligned}
$$

Thanks to $(80)$ and (82), we get

$$
\begin{aligned}
\left|\tilde{u}_{i, j}^{h}-\tilde{u}_{i-1, j}^{h}\right| & \leq\left|f^{h}(i)+\hat{g}^{h}(j)\right|+\left|f^{h}(i-1)+\hat{g}^{h}(j)\right| \\
& \leq\left|f^{h}(i)-f^{h}(i-1)\right| .
\end{aligned}
$$

In the second case,

$$
\hat{f}^{h}(i-1)+\hat{g}^{h}(j)<0 .
$$

If $\hat{f}^{h}(i-1)=\hat{f}^{h}(i)$, we have immediately

$$
\left|\tilde{u}_{i, j}^{h}-\tilde{u}_{i-1, j}^{h}\right| \leq\left|f^{h}(i)-f^{h}(i-1)\right| .
$$

If $\hat{f}^{h}(i-1)>\hat{f}^{h}(i)$, then, we have (82), and

$$
\begin{aligned}
\tilde{u}_{i, j}^{h}-\tilde{u}_{i-1, j}^{h} & =f^{h}(i)+g^{h}(j)-2 \hat{f}^{h}(i)-2 \hat{g}^{h}(j)-f^{h}(i-1)-g^{h}(j)+2 \hat{f}^{h}(i-1)+2 \hat{g}^{h}(j) \\
& =2 \hat{f}^{h}(i-1)-f^{h}(i)-f^{h}(i-1),
\end{aligned}
$$

and, thanks to (82) we have

$$
\left|\tilde{u}_{i, j}^{h}-\tilde{u}_{i-1, j}^{h}\right| \leq\left|f^{h}(i)-f^{h}(i-1)\right| .
$$

From (83), (84) and (85), we deduce

$$
\left|\tilde{u}_{i, j}^{h}-\tilde{u}_{i-1, j}^{h}\right| \leq\left|w_{i, j}^{h}-w_{i-1, j}^{h}\right|=\frac{1}{2}\left|\int_{(i-1) h}^{i h}\left(u_{0 x}+u_{1}\right)\left(x^{\prime}\right) d x^{\prime}\right| .
$$

The proof of (72) is analogous.

We can deduce from (71) and (72) an energy inequality. Let $i_{0}, j_{0}$ be given such that $-n \leq i_{0}, j_{0} \leq n$ and $i_{0}+j_{0} \geq 0$. Then we have

$$
\begin{aligned}
\sum_{i=-j_{0}+1}^{i_{0}} \frac{1}{h}\left|\tilde{u}_{i, j_{0}}^{h}-\tilde{u}_{i-1, j_{0}}^{h}\right|+\sum_{j=-i_{0}+1}^{j_{0}} \frac{1}{h} \mid \tilde{u}_{i_{0}, j}^{h} & -\left.\tilde{u}_{i_{0}, j-1}^{h}\right|^{2} \\
& \leq \frac{1}{2} \int_{-j_{0} h}^{i_{0} h}\left[\left|u_{0 x}\right|^{2}\left(x^{\prime}\right)+\left|u_{1}\right|^{2}\left(x^{\prime}\right)\right] d x^{\prime} .
\end{aligned}
$$

3.2. A numerical scheme for the string with fixed ends and a constant obstacle. We shall use here the inductive construction of $\$ 2.3$, which we discretize.

Let $u_{0}$ and $u_{1}$ be given on $[0, L]$, and let

$$
\alpha=K^{2} /\left[\int_{0}^{L}\left(\left|\frac{d u_{0}}{d x}\right|^{2}+\left|u_{1}\right|^{2}\right) d x\right],
$$

where the obstacle is $\varphi(x)=-K<0$.

Let $n$ be an even integer, and let the step be $h=L / n$; let $n_{0}$ be the largest integer such that $n_{0} h \leq \alpha$. 
We discretize the initial data as in (64) for $0 \leq p \leq n$, and we extend them as periodic and odd functions:

$$
u_{r}^{h}(x)= \begin{cases}-u_{r}^{h}(-x) & \text { for }-n_{0} h \leq x \leq 0, \quad r=0,1 \\ -u_{r}^{h}(2 L-x) & \text { for } n h \leq x \leq\left(n+n_{0}\right) h, \quad r=0,1\end{cases}
$$

We define $w^{0, h}$ by

$$
\begin{aligned}
& w^{0, h}(x, 0)=u_{0}^{h}(x), \quad-n_{0} h \leq x \leq\left(n+n_{0}\right) h, \\
& \frac{\partial w^{0, h}}{\partial t}(x, 0)=u_{1}^{h}(x), \quad-n_{0} h \leq x \leq\left(n+n_{0}\right) h, \\
& \square w^{0, h}=0 \quad \text { in } T_{(n / 2) h,\left(n / 2+n_{0}\right) h}^{-},
\end{aligned}
$$

and let

$$
\tilde{w}_{i, j}^{0, h}=w^{0, h}\left(\frac{i-j}{2} h, \frac{i+j}{2} h\right)
$$

Let

$$
\tilde{u}_{i, j}^{h}=\tilde{w}_{i, j}^{0, h}+2 \sup \left\{\left(\tilde{w}_{i, j}^{0, h}+K\right)^{-}: i^{\prime} \leq i, j^{\prime} \leq j, i^{\prime}+j^{\prime} \geq 0\right\}
$$

where $i \leq n+n_{0}, j \leq n_{0}, i+j \geq 0$.

Let us define a subset $R^{m, h}$ of $\mathbb{Z} \times \mathbb{Z}$ by

$$
\begin{gathered}
R^{m, h}=\left[n+(2 m-1) n_{0}, n+(2 m+1) n_{0}\right] \times\left[-n+(2 m-1) n_{0},(2 m-1) n_{0}\right] \\
\cup\left[(2 m-1) n_{0}, n+(2 m+1) n_{0}\right] \times\left[(2 m-1) n_{0},(2 m+1) n_{0}\right] .
\end{gathered}
$$

The region $R^{m, h}$ is the discretized equivalent (in $i, j$ coordinates) of the region $R^{m}$ defined by (59). We define $\tilde{w}^{m, h}$ on the lower boundary of $R^{m, h}$ by

$$
\tilde{w}_{i, j}^{m, h}= \begin{cases}\tilde{u}_{i, j}^{n} & \text { for } i=n+(2 m-1) n_{0},-n+(2 m-1) n_{0} \leq j \leq(2 m-1) n_{0}, \\ \tilde{u}_{i, j}^{h} & \text { for } j=(2 m-1) n_{0},(2 m-1) n_{0} \leq i \leq n+(2 m-1) n_{0}, \\ \tilde{w}_{j, i}^{m, h} & \text { for } i=(2 m-1) n_{0},(2 m-1) n_{0} \leq j \leq(2 m+1) n_{0}, \\ \tilde{w}_{n+j,-n+i}^{m, h} & \text { for }(2 m-1) n_{0}+n \leq i \leq(2 m+1) n_{0}+n, j=-n+(2 m-1) n,\end{cases}
$$

and in $R^{m, h}$, we have

$$
\tilde{w}_{i, j}^{m, h}=\tilde{w}_{i-1, j}^{m, h}+\tilde{w}_{i, j-1}^{m, h}-\tilde{w}_{i-1, j-1}^{m, h} \quad \text { for }(i, j),(i-1, j-1) \quad \text { in } R^{m, h} .
$$

Then, we shall define $\tilde{u}_{i, j}^{h}$ on $R^{m, h} \cap\{(i, j): 0 \leq(i-j) / 2 \leq n\}$ by

$$
\tilde{u}_{i, j}^{h}=\tilde{w}_{i, j}^{m, h}+2 \sup \left\{\left(\tilde{w}_{i^{\prime}, j^{\prime}}^{m, h}+K\right)^{-}: i^{\prime} \leq i, j^{\prime} \leq j \text { and }\left(i^{\prime}, j^{\prime}\right) \in R^{m, h}\right\} .
$$

Of course (94) is the discretization of (63).

THEOREM 9. Let $u^{h}$ be defined by (93), and let $u$ be the solution of $\left(\mathrm{P}_{f}\right)$ on $[0, L]$ with obstacle $-K$. Then

$$
\max _{i, j}\left|\tilde{u}_{i, j}^{h}-u\left(\frac{i-j}{2} h, \frac{i+j}{2} h\right)\right| \leq C^{m+1} \sqrt{h}
$$


for $(i, j)$ in the region $R^{m, h}$ defined by (91), where $C$ depends only on the initial conditions. Moreover, we have the following bounds on the (approximate) characteristic derivatives:

$$
\begin{aligned}
& \left|\tilde{u}_{i, j}^{h}-\tilde{u}_{i-1, j}^{h}\right| \leq \frac{1}{2}\left|\int_{(i-1) h}^{i h}\left(u_{0 x}+u_{1}\right)\left(x^{\prime}\right) d x^{\prime}\right|, \\
& \left|\tilde{u}_{i, j}^{h}-\tilde{u}_{i, j-1}^{h}\right| \leq \frac{1}{2}\left|\int_{-j h}^{(-j+1) h}\left(u_{0 x}-u_{1}\right)\left(x^{\prime}\right) d x^{\prime}\right|,
\end{aligned}
$$

if $u_{0}$ and $u_{1}$ are extended to all $\mathbb{R}$ by periodicity and imparity.

Proof. We shall replace the number $\alpha$ defined in (87) by $n_{0} h$; for this new value of $\alpha$, we can perform the construction of the solution of $P_{f}$ as in 2.3, and we shall compare $w^{m}$ and $\tilde{w}_{i, j}^{m, h}$ on the regions $R^{m}$ and $R^{m, h}$.

Thanks to Theorem 8, the relation (95) is verified for $m=0$ and $C \geq\left(2 \int_{-\alpha}^{L+\alpha}\left(u_{0 x}^{2}+\right.\right.$ $\left.\left.u_{1}^{2}\right) d x\right)^{1 / 2}$, and the relation (96) is satisfied in $R_{0}$.

Suppose that for a certain constant $C,(95)$ and (96) are satisfied in $R^{m-1, h}$.

Then we have

$$
\left|\tilde{w}_{i, j}^{m, h}-w^{m}\left(\frac{i-j}{2} h, \frac{i+j}{2} h\right)\right| \leq C^{m} \sqrt{h}
$$

for $i, j$ on the lower boundary of $R^{m, h}$ which is the upper boundary of $R^{m-1, h}$.

Then we have

$$
\left|\tilde{w}_{i, j}^{m, h}-w^{m}\left(\frac{i-j}{2} h, \frac{i+j}{2} h\right)\right| \leq 5 C^{m} \sqrt{h} \quad \text { in } R^{m, h},
$$

because $\tilde{w}_{i, j}^{m, h}$ (respectively $\left.w^{m}(((i-j) / 2) h,((i+j) / 2) h)\right)$ is the sum of at most five terms $\tilde{w}_{i^{\prime}, j^{\prime}}^{m}$ (respectively $\left.w^{m}\left(\left(\left(i^{\prime}-j^{\prime}\right) / 2\right) h,\left(\left(i^{\prime}+j^{\prime}\right) / 2\right) h\right)\right)$ with $i^{\prime}, j^{\prime}$ on the lower boundary of $R^{m, h}$ (respectively $\left.\left(\left(\left(i^{\prime}-j^{\prime}\right) / 2\right) h,\left(\left(i^{\prime}+j^{\prime}\right) / 2\right) h\right)\right)$ on the lower boundary of $\left.R^{m}\right)$.

If we now evaluate the difference $\tilde{w}_{i, j}^{m, h}-w^{m}\left(x^{\prime}, t^{\prime}\right)$ when $\left(x^{\prime}, t^{\prime}\right)$ is in the characteristic square centered on $((i-j) / 2) h,((i+j) / 2) h$ with sides of length $h \sqrt{2}$, we have

$$
\left|\tilde{w}_{i, j}^{m, h}-w^{m}\left(x^{\prime}, t^{\prime}\right)\right| \leq 5 C^{m} \sqrt{h}+\left|w^{m}\left(\frac{i-j}{2} h, \frac{i+j}{2} h\right)-w^{m}\left(x^{\prime}, t^{\prime}\right)\right|,
$$

but we have for $P_{f}$ the equivalent of (48), i.e.,

$$
\begin{aligned}
& \left|\frac{\partial u}{\partial \xi}(x, t)\right|=\left|\frac{\partial u}{\partial \xi}(x+t, 0)\right|=\left|\frac{1}{\sqrt{2}}\left(u_{0 x}+u_{1}\right)(x-t, 0)\right|, \\
& \left|\frac{\partial u}{\partial \eta}(x, t)\right|=\left|\frac{\partial u}{\partial \eta}(x-t, 0)\right|=\left|\frac{1}{\sqrt{2}}\left(u_{0 x}-u_{1}\right)(x-t, 0)\right|,
\end{aligned}
$$

if $u_{0}$ and $u_{1}$ are extended to all of $\mathbb{R}$ by imparity and periodicity.

Therefore

$$
\begin{aligned}
& \left|\frac{\partial w^{m}}{\partial \xi}(x, t)\right|=\frac{1}{\sqrt{2}}\left|\left(u_{0 x}+u_{1}\right)(x+t, 0)\right|, \\
& \left|\frac{\partial w^{m}}{\partial \eta}(x, t)\right|=\frac{1}{\sqrt{2}}\left|\left(u_{0 x}-u_{1}\right)(x-t, 0)\right| .
\end{aligned}
$$


Relation (100) allows us to evaluate $w^{m}(((i-j) / 2) h,((i+j) / 2) h)-w^{m}\left(x^{\prime}, t^{\prime}\right)$,

$$
\left|w^{m}\left(\frac{i-j}{2} h, \frac{i+j}{2} h\right)-w^{m}\left(x^{\prime}, t^{\prime}\right)\right| \leq \sqrt{2 h}\left(\int_{-\alpha}^{L+\alpha}\left(u_{0 x}^{2}+u_{1}^{2}\right) d x\right)^{1 / 2} \text {. }
$$

Let us denote by $E$ the number, which has the dimension of an energy:

$$
E=\int_{-\alpha}^{L+\alpha}\left(u_{0 x}^{2}+u_{1}^{2}\right) d x
$$

Gathering relations (97), (99) and (101), we obtain:

$$
\left|\tilde{u}_{i, j}^{h}-u\left(\frac{i-j}{2} h, \frac{i+j}{2} h\right)\right| \leq\left(15 C^{m}+2 \sqrt{2 E}\right) \sqrt{h} .
$$

Therefore, if we choose $C=15+2 \sqrt{2} E$, we have

$$
15 C^{m}+2 \sqrt{2 E} \leq C^{m+1} .
$$

The proof of (96) is immediate.

Remark. For $(i, j)$ in $R^{m, h}$, we have

$$
\frac{i+j}{2} \geq(2 m-1) n_{0}
$$

and thus

$$
1+m \leq\left(\left(\frac{i+j}{2} h\right) / 2 n_{0} h\right)+\frac{3}{2} .
$$

Therefore, if $(((i-j) / 2) h,((i+j) / 2) h)$ converges to $(x, t)$ as $h$ goes to zero, we have from (95):

$$
\left|\tilde{u}_{i, j}^{h}-u\left(\frac{i-j}{2} h, \frac{i+j}{2} h\right)\right| \leq C_{1}^{t / 2 \alpha} C_{1}^{3 / 2} \sqrt{h}
$$

for all $C_{1}>C$, and for all $h$ small enough.

4. Regularity in spaces of functions of locally bounded variation. This section is dedicated to proving the following result of regularity for an arbitrary concave obstacle $\varphi$. that

$$
\frac{d u_{0}}{d x} \text { and } u_{1} \text { are locally of bounded variation. }
$$

Suppose that $u_{0}$ and $u_{1}$ satisfy the compatibility condition (22), and that the obstacle is concave.

Then for all $\eta$, the function

$$
\xi \rightarrow \frac{\partial \tilde{u}}{\partial \xi}(\xi, \eta)
$$

defined on $[-\eta,+\infty)$ is locally of bounded variation, and analogously, for all $\xi$ the function

$$
\eta \rightarrow \frac{\partial \tilde{u}}{\partial \eta}(\xi, \eta)
$$

defined on $[-\xi,+\infty)$ is locally of bounded variation. 
Proof. We retain the notation of $\$ 2.1$ :

(35)

$$
\begin{gathered}
\left.U=\{x: w(x, \tau(x))>0\}=\bigcup_{i}\right] a_{i}, b_{i}[; \\
\xi_{i}=\frac{a_{i}+\tau\left(a_{i}\right)}{\sqrt{2}}, \quad \xi_{i}^{\prime}=\frac{b_{i}+\tau\left(b_{i}\right)}{\sqrt{2}}, \\
\eta_{i}=\frac{-a_{i}+\tau\left(a_{i}\right)}{\sqrt{2}}, \quad \eta_{i}^{\prime}=\frac{-b_{i}+\tau\left(b_{i}\right)}{\sqrt{2}} ; \\
Y(\xi)= \begin{cases}\eta_{i} & \text { if } \xi \in\left[\xi_{i}, \xi_{i}^{\prime}\right), \\
{\left[\eta_{i}^{\prime}, \eta_{i}\right]} & \text { if } \xi=\xi_{i}^{\prime},\end{cases} \\
C=\left(\bigcup_{i}\left[\xi_{i}, \xi_{i}^{\prime}\right]\right)^{c} .
\end{gathered}
$$

We have the following representation of the solution:

$$
\tilde{u}(\xi, \eta)= \begin{cases}f(\xi)+g(\eta) & \text { for } \eta \leq Y(\xi), \\ \hat{f}(\xi)+\hat{g}(\eta) & \text { for } \eta \geq Y(\xi),\end{cases}
$$

with the transmission conditions:

$$
\begin{gathered}
f(\xi)+g(Y(\xi))=\hat{f}(\xi)+\hat{g}(Y(\xi))=\varphi\left[\frac{\xi-Y(\xi)}{\sqrt{2}}\right] \\
f^{\prime}(\xi)+g^{\prime}(Y(\xi))=\left[\hat{f}^{\prime}(\xi)+\hat{g}^{\prime}(Y(\xi))\right] \\
\text { if } Y \text { is one-valued and } 0>Y^{\prime}(\xi)>-\infty .
\end{gathered}
$$

If we differentiate (104) with respect to $\xi$ on $C$, we get

(106) $f^{\prime}(\xi)+Y^{\prime}(\xi) g^{\prime}(Y(\xi))=\hat{f}^{\prime}(\xi)+Y^{\prime}(\xi) \hat{g}^{\prime}(Y(\xi))=\frac{1}{\sqrt{2}} \varphi^{\prime}\left[\frac{\xi-Y(\xi)}{\sqrt{2}}\right]\left(1-Y^{\prime}(\xi)\right)$

(notice that $Y$ is decreasing on $C$, and therefore almost everywhere differentiable). For $Y^{\prime}(\xi)=0$, we deduce from $(106)$ that

$$
f^{\prime}(\xi)=\hat{f}^{\prime}(\xi)=\frac{1}{\sqrt{2}} \varphi^{\prime}(\xi-Y(\xi))
$$

For $0>Y^{\prime}(\xi)>-\infty$, we deduce from $(105)$ and $(106)$ that $\hat{f}^{\prime}(\xi)+f^{\prime}(\xi)=\sqrt{2} \varphi^{\prime}(\xi-$ $Y(\xi)$ ), which contains (107). Therefore, we have

$$
\hat{f}^{\prime}(\xi)+f^{\prime}(\xi)=\sqrt{2} \varphi^{\prime}(\xi-Y(\xi)) \text { a.e. on } C,
$$

and differentiating (104) on $C^{c}$,

$$
\hat{f}^{\prime}(\xi)=f^{\prime}(\xi) \text { a.e. on } C^{c} .
$$

Let us denote by $h$ the function

$$
h(\xi)=\frac{\partial u}{\partial \xi}(\xi, \eta)
$$


where $\eta$ is fixed throughout the end of this proof, and let $\xi_{0}=\sup \left\{\xi^{\prime} \in X(\eta)\right\}$. Then, for $\xi \leq \xi_{0}, h(\xi)=f(\xi)$ and $\xi_{0}$ does not belong to any interval $\left(\xi_{i}, \xi_{i}^{\prime}\right)$.

To evaluate the total variation of $h$ on a given bounded interval $I=[a, b]$, we have to estimate

$$
\begin{aligned}
& T V(h: I)=T V\left(h ; I \cap\left(-\infty, \xi_{0}\right)\right)+T V\left(h ; C \cap\left(\xi_{0},+\infty\right)\right) \\
&+ T V\left(h ; C^{c} \cap\left(\xi_{0},+\infty\right)\right)+\left|h\left(\xi_{0}+0\right)-h\left(\xi_{0}-0\right)\right| \\
&+\sum_{\left\{i: \xi_{0} \leq \xi_{i} \leq b\right\}}\left[\left|h\left(\xi_{i}^{\prime}+0\right)-h\left(\xi_{i}^{\prime}-0\right)\right|+\left|h\left(\xi_{i}+0\right)-h\left(\xi_{i}-0\right)\right|\right] .
\end{aligned}
$$

According to (108) and (109), we have:

$$
\begin{aligned}
T V\left(h ; I \cap\left(-\infty, \xi_{0}\right)\right)+T V( & \left.h ; C \cap\left(\xi_{0},+\infty\right)\right)+T V\left(h ; C^{c} \cap\left(\xi_{0},+\infty\right)\right) \\
\leq & T V(f ; I)+T V\left(\sqrt{2} \varphi^{\prime}\left(\frac{\xi-Y(\xi)}{\sqrt{2}}\right)-f(\xi) ; I\right) .
\end{aligned}
$$

By hypothesis, $\varphi^{\prime \prime}$ is positive, therefore $\varphi^{\prime}$ is increasing; as $\xi \mapsto(\xi-Y(\xi)) / \sqrt{2}$ is increasing, the right-hand side of (112) is bounded.

The term $\left|h\left(\xi_{0}+0\right)-h\left(\xi_{0}-0\right)\right|$ is bounded, because (102) ensures that $f$, and therefore $\hat{f}$, is locally bounded.

The remaining term in (111) is the sum

$$
\sum_{\left\{i: \xi_{0} \leq \xi_{i} \leq b\right\}}\left[\left|h\left(\xi_{i}^{\prime}+0\right)-h\left(\xi_{i}^{\prime}-0\right)\right|+\left|h\left(\xi_{i}+0\right)-h\left(\xi_{i}-0\right)\right|\right],
$$

which could possibly contain an infinite number of terms. Using (108) and (109), we can write the terms of (113) as

$$
\left|f\left(\xi_{i}+0\right)+f\left(\xi_{i}-0\right)-\sqrt{2} \varphi^{\prime}\left(\frac{\xi_{i}-\eta_{i}}{\sqrt{2}}-0\right)\right|+\left|f\left(\xi_{i}^{\prime}+0\right)+f\left(\xi_{i}^{\prime}-0\right)-\sqrt{2} \varphi^{\prime}\left(\frac{\xi_{i}^{\prime}-\eta_{i}^{\prime}}{\sqrt{2}}+0\right)\right| .
$$

But we have the following inequalities, deduced from the definition of the line of influence and of the intervals $\left[\xi_{i}, \xi_{i}^{\prime}\right]$ :

$$
\begin{aligned}
& f\left(\xi_{i}-0\right)-\frac{1}{\sqrt{2}} \varphi^{\prime}\left(\frac{\xi_{i}-\eta_{i}}{\sqrt{2}}-0\right)=a_{i}^{-} \leq 0, \\
& f\left(\xi_{i}+0\right)-\frac{1}{\sqrt{2}} \varphi^{\prime}\left(\frac{\xi_{i}-\eta_{i}}{\sqrt{2}}+0\right)=a_{i}^{+} \geq 0, \\
& f\left(\xi_{i}^{\prime}-0\right)-\frac{1}{\sqrt{2}} \varphi^{\prime}\left(\frac{\xi_{i}^{\prime}-\eta_{i}^{\prime}}{\sqrt{2}}-0\right)=b_{i}^{-} \leq 0, \\
& f\left(\xi_{i}^{\prime}+0\right)-\frac{1}{\sqrt{2}} \varphi^{\prime}\left(\frac{\xi_{i}^{\prime}-\eta_{i}^{\prime}}{\sqrt{2}}+0\right)=b_{i}^{+} \leq 0 .
\end{aligned}
$$


We can estimate (114) by

$$
\begin{aligned}
\left|a_{i}^{+}+a_{i}^{-}\right|+\left|b_{i}^{+}+b_{i}^{-}\right|+\frac{1}{\sqrt{2}}\left[\left|\varphi^{\prime}\left(\frac{\xi_{i}-\eta_{i}}{\sqrt{2}}+0\right)-\varphi^{\prime}\left(\frac{\xi_{i}-\eta_{i}}{\sqrt{2}}-0\right)\right|\right. \\
\left.+\left|\varphi^{\prime}\left(\frac{\xi_{i}^{\prime}-\eta_{i}^{\prime}}{\sqrt{2}}+0\right)-\varphi^{\prime}\left(\frac{\xi_{i}^{\prime}-\eta_{i}^{\prime}}{\sqrt{2}}-0\right)\right|\right]
\end{aligned}
$$

But

$$
\left|a_{i}^{+}+a_{i}^{-}\right|+\left|b_{i}^{+}+b_{i}^{-}\right| \leq\left|a_{i}^{+}+a_{i}^{-}\right|+\left|b_{i}^{+}-a_{i}^{+}\right|+\left|a_{i}^{+}+a_{i}^{-}\right|+\left|a_{i}^{-}-b_{i}^{-}\right|,
$$

and using the sign conditions (115),

$$
\begin{aligned}
\left|a_{i}^{+}+a_{i}^{-}\right|+\left|b_{i}^{+}+b_{i}^{-}\right| & \leq 2\left|a_{i}^{+}+a_{i}^{-}\right|+\left|b_{i}^{+}-a_{i}^{+}\right|+\left|b_{i}^{-}+a_{i}^{-}\right| \\
& \leq 4 T V\left(f(\xi)-\frac{1}{\sqrt{2}} \varphi^{\prime}\left(\frac{\xi-y(\xi)}{\sqrt{2}}\right) ;\left[\xi_{i}, \xi_{i}^{\prime}\right]\right) .
\end{aligned}
$$

Carrying (117) and (116) into (113), we obtain:

$$
\begin{aligned}
\sum_{\left\{i: \xi_{0} \leq \xi_{i} \leq b\right\}}\left[\mid h\left(\xi_{i}^{\prime}+0\right)\right. & \left.-h\left(\xi_{i}^{\prime}-0\right)|+| h\left(\xi_{i}+0\right)-h\left(\xi_{i}-0\right) \mid\right] \\
& \leq 4 T V\left(f ;\left[\xi_{0}, \xi_{0}^{\prime}\right]\right)+5 T V\left(\frac{1}{\sqrt{2}} \varphi^{\prime}\left(\frac{\xi-y(\xi)}{\sqrt{2}}\right) ;\left[\xi_{0}, \xi_{0}^{\prime}\right]\right) .
\end{aligned}
$$

Here, $\xi_{0}^{\prime}=\sup \left\{\xi_{i}^{\prime}: \xi_{i}^{\prime} \leq b\right\}$. The same argument holds for the other characteristic derivative. The proof of Theorem 10 is complete; notice that we have proved, in fact, that locally, $T V((\partial \tilde{u} / \partial \xi)(\cdot, \eta), I)$ is a bounded function of $\eta$, for all bounded $I$.

Remark 11. It is not true that under hypothesis $(102),(\partial u / \partial \xi)(\cdot, t)$ or $(\partial u / \partial \xi)(\cdot, t)$ are of bounded variation for all $t$.

To see it, let us consider the following example. Let

$$
w(x, t)= \begin{cases}A-t-a(x+t)^{4} \sin \frac{1}{x+t} & \text { if }|x+t| \leq b, \\ A-t & \text { if }|x+t| \geq b .\end{cases}
$$

We choose $b$ such that $\sin (1 / b)=0$, and $a$ such that the curve

$$
t=A-a(x+t)^{4} \sin \frac{1}{x+t}
$$

always has a slope less than 1 , for $|x+t| \leq b$. For this purpose, we differentiate (120) with respect to $x$ :

$$
t^{\prime}=4 a\left(1+t^{\prime}\right)(x+t)^{3} \sin \frac{1}{x+t}-a(x+t)^{2} \cos \frac{1}{x+t} \cdot\left(1+t^{\prime}\right)
$$

and so,

$$
\left|t^{\prime}\right| \leq a \frac{4 b^{3}+b^{2}}{1-a\left(4 b^{3}+b^{2}\right)} .
$$

Clearly $\left|t^{\prime}\right|$ can be made smaller than 1 if $a$ is sufficiently small. 
Then we choose $A$ large enough to have

$$
w(x, 0)=A-a x^{4} \sin \frac{1}{x}>0 \text { for }|x| \leq b .
$$

Obviously, $d u_{0} / d x=w_{x}(x, 0)$ and $u_{1}=w_{t}(x, 0)$ are locally of bounded variation.

Thanks to (121), the line of influence is given by (120). We shall now see that $(\partial u / \partial \eta)(\cdot, A)$ is not of bounded variation. The straight line $t=A$ crosses the line of influence infinitely many times, at the points

$$
x=\frac{1}{n \pi}-A \quad \text { for }\left|\frac{1}{n \pi}\right|<b, \quad n \in \mathbb{Z},
$$

and we have

$$
\frac{\partial u}{\partial \eta}(x, A)=\left\{\begin{array}{rll}
-1 & \text { if } x \in\left(\frac{1}{(2 k+2) \pi}-A, \frac{1}{(2 k+1) \pi}-A\right), & k>0 \\
& \text { or if } x \in\left(\frac{1}{(2 k+1)}-A, \frac{1}{(2 k+2)}-A\right), & k<0, \\
+1 & \text { if } x \in\left(\frac{1}{(2 k+1) \pi}-A, \frac{1}{2 k \pi}-A\right), & k>0 \\
& \text { or if } x \in\left(\frac{1}{2 k \pi}-A, \frac{1}{(2 k+1) \pi}-A\right), & k<0 .
\end{array}\right.
$$

This function is not of bounded variation on any interval containing zero.

5. Convergence of the penalty method.

5.1. Weak convergence. This paragraph is dedicated to a general (and unfortunately coarse!) study of the penalized problem

$$
\begin{aligned}
& \square u_{\lambda}-\frac{1}{\lambda}\left(u_{\lambda}-\varphi\right)^{-}=0, \\
& u_{\lambda}(x, 0)=u_{0}(x), \\
& \frac{\partial u_{\lambda}}{\partial t}(x, 0)=u_{1}(x),
\end{aligned}
$$

where $r^{-}=\sup (-r, 0)$, and $\varphi$ is an arbitrary continuous function of $x$, and $u_{0}, u_{1}$ satisfy the compatibility condition (22). The parameter $\lambda$ is positive, and will tend to zero.

Let us mention that (122) always possesses a unique solution; to see this, it is enough to write (122) in the form of an integral equation, and to use Picard iterations.

Proposition 12. We have the following estimates for the solution $u_{\lambda}$ of (122):

$$
\begin{aligned}
\int_{a}^{b}\left[\left|\frac{\partial u_{\lambda}}{\partial t}(x, \sigma(x))\right|^{2}+\left|\frac{\partial u_{\lambda}}{\partial x}(x, \sigma(x))\right|^{2}+2\left(\frac{\partial u_{\lambda}}{\partial t} \frac{\partial u_{\lambda}}{\partial x}\right)\right. & \left.(x, \sigma(x)) \sigma^{\prime}(x)\right] d x \\
& \leq \int_{a}^{b}\left(\left|\frac{d u_{0}}{d x}\right|^{2}+\left|u_{1}\right|^{2}\right) d x
\end{aligned}
$$

for all Lipschitz continuous $\sigma$ with Lipschitz constant 1 such that $\sigma>0$ on $(a, b), \sigma(a)=$ $\sigma(b)=0$;

$$
\int_{T_{x, t}^{-}} \frac{1}{\lambda}\left(u_{\lambda}\left(x^{\prime}, t^{\prime}\right)-\varphi\left(x^{\prime}\right)\right)^{-} d x^{\prime} d t^{\prime} \leq C\left(x, t, u_{0}, u_{1}\right)
$$

where $C$ does not depend on $\lambda$. 
Proof. (i) Estimate (123). We have the identity

$$
\begin{aligned}
& \left(\square u_{\lambda}-\frac{1}{\lambda}\left(u_{\lambda}-\varphi\right)^{-}\right) \frac{\partial u_{\lambda}}{\partial t} \\
& =\frac{\partial}{\partial x}\left(-\frac{\partial u_{\lambda}}{\partial t} \frac{\partial u_{\lambda}}{\partial x}\right)+\frac{1}{2} \frac{\partial}{\partial t}\left(\left|\frac{\partial u_{\lambda}}{\partial x}\right|^{2}+\left|\frac{\partial u_{\lambda}}{\partial x}\right|^{2}+\frac{1}{\lambda}\left(\left(u_{\lambda}-\varphi\right)^{-}\right)^{2}\right)=0 .
\end{aligned}
$$

Integrating on the region $\{(x, t): a \leq x \leq b$ and $0 \leq t \leq \sigma(x)\}$, we obtain the identity

$$
\begin{aligned}
& \int_{a}^{b}\left[\left|\frac{\partial u_{\lambda}}{\partial t}(x, \sigma(x))\right|^{2}+\left|\frac{\partial u_{\lambda}}{\partial x}(x, \sigma(x))\right|^{2}\right. \\
& \left.+2 \sigma^{\prime}(x)\left(\frac{\partial u_{\lambda}}{\partial t} \frac{\partial u_{\lambda}}{\partial x}\right)(x, \sigma(x))+\frac{1}{\lambda}\left(\left(u_{\lambda}(x, \sigma(x))-\varphi(x)\right)^{-}\right)^{2}\right] d x \\
& =\int_{a}^{b}\left(\left|\frac{d u_{0}}{d x}\right|^{2}+\left|u_{1}\right|^{2}\right) d x,
\end{aligned}
$$

noting that $\left(u_{\lambda}(x, 0)-\varphi(x)\right)^{-}=0$ for all $x$. From here, (123) is immediate.

(ii). Estimate (124).

We integrate $\square u_{\lambda}=(1 / \lambda)\left(u_{\lambda}-\varphi\right)^{-}$on the backward cone $T_{x, t}^{-}$:

$$
\begin{aligned}
\int_{T_{x, t}} \square & u_{\lambda} d x^{\prime} d t^{\prime} \\
= & \int_{x-t}^{x+t}\left(\frac{\partial u_{\lambda}}{\partial t}\left(x^{\prime}, t-\left|x-x^{\prime}\right|\right)-u_{1}\left(x^{\prime}, 0\right)\right) d x^{\prime} \\
& \quad-\int_{0}^{t}\left(\frac{\partial u_{\lambda}}{\partial x}\left(x+t-t^{\prime}, t^{\prime}\right)-\frac{\partial u_{\lambda}}{\partial x}\left(x-t+t^{\prime}\right)\right) d t^{\prime} \\
= & \int_{x-t}^{x}\left[\frac{\partial u_{\lambda}}{\partial t}\left(x^{\prime}, t-\left|x-x^{\prime}\right|\right)+\frac{\partial u_{\lambda}}{\partial x}\left(x^{\prime}, t-\left|x-x^{\prime}\right|\right)\right] d x^{\prime} \\
& \quad+\int_{x}^{x+t}\left[\frac{\partial u_{\lambda}}{\partial t}\left(x^{\prime}, t-\left|x-x^{\prime}\right|\right)-\frac{\partial u_{\lambda}}{\partial x}\left(x^{\prime}, t-\left|x-x^{\prime}\right|\right)\right] d x^{\prime}-\int_{x-t}^{x+t} u_{1}\left(x^{\prime}\right) d x^{\prime} .
\end{aligned}
$$

Let $\sigma\left(x^{\prime}\right)=t-\left|x-x^{\prime}\right|$. Then

$$
\begin{aligned}
& \int_{T_{x, t}} \square u_{\lambda}\left(x^{\prime}, t^{\prime}\right) d x^{\prime} d t^{\prime} \\
& \quad \leq \int_{x-t}^{x+t}\left[\frac{\partial u_{\lambda}}{\partial t}\left(x^{\prime}, \sigma\left(x^{\prime}\right)\right)+\sigma^{\prime}\left(x^{\prime}\right) \frac{\partial u_{\lambda}}{\partial x}\left(x^{\prime}, \sigma\left(x^{\prime}\right)\right)\right] d x^{\prime}+\int_{x-t}^{x+t}\left|u_{1}\left(x^{\prime}\right)\right| d x^{\prime},
\end{aligned}
$$

and using the Schwarz inequality and (123), we obtain

$$
\begin{aligned}
\int_{T_{x, t}^{-}} \frac{1}{\lambda}\left(u_{\lambda}-\varphi\right)^{-} d x^{\prime} d t^{\prime} & \leq\left[\int_{x-t}^{x+t}\left[\frac{\partial u_{\lambda}}{\partial t}\left(x^{\prime}, \sigma\left(x^{\prime}\right)\right)+\sigma^{\prime}\left(x^{\prime}\right) \frac{\partial u_{\lambda}}{\partial x}\left(x^{\prime}, \sigma\left(x^{\prime}\right)\right)\right]^{2} d x^{\prime}\right]^{1 / 2} \sqrt{2 t} \\
& +\left(\int_{x-t}^{x+t}\left|u_{1}\left(x^{\prime}\right)\right|^{2} d x^{\prime}\right)^{1 / 2} \sqrt{2 t} \\
& \leq 2 \sqrt{2 t}\left(\int_{x-t}^{x+t}\left(\mid\left(\left.u_{1}\left(x^{\prime}\right)\right|^{2}+\left|\frac{d u_{0}}{d x}\left(x^{\prime}\right)\right|^{2}\right) d x^{\prime}\right)^{1 / 2}\right.
\end{aligned}
$$


We need definitions of left and right traces of the characteristic derivatives of a function $u$.

The following results were proved in [5]: let $u$ be in $V$ (cf. Def. (17)), such that $\square u$ is a positive measure. Then the function

$$
\left.\eta \mapsto \frac{\partial \tilde{u}}{\partial \xi}(\xi, \eta)\right|_{[a, b]}
$$

is increasing from $[-a, \infty)$ to $L^{2}(a, b)$ for all $a, b$, and similarly

$$
\left.\xi \mapsto \frac{\partial \tilde{u}}{\partial \eta}(\xi, \eta)\right|_{[c, d]}
$$

is increasing from $[-c, \infty)$ to $L^{2}(c, d)$ for all $c, d$.

We define

$$
\begin{aligned}
& \frac{\partial \tilde{u}^{r}}{\partial \xi}(\xi, \eta)=\lim _{h \downarrow 0} \frac{\partial \tilde{u}}{\partial \xi}(\xi, \eta+h), \\
& \frac{\partial \tilde{u}^{l}}{\partial \xi}(\xi, \eta)=\lim _{h \downarrow 0} \frac{\partial \tilde{u}}{\partial \xi}(\xi, \eta-h), \\
& \frac{\partial \tilde{u}^{r}}{\partial \eta}(\xi, \eta)=\lim _{h \downarrow 0} \frac{\partial \tilde{u}}{\partial \eta}(\xi+h, \eta), \\
& \frac{\partial \tilde{u}^{l}}{\partial \eta}(\xi, \eta)=\lim _{h \downarrow 0} \frac{\partial \tilde{u}}{\partial \eta}(\xi-h, \eta) .
\end{aligned}
$$

The functions $\partial \tilde{u}^{r} / \partial \xi$ and $\partial \tilde{u}^{l} / \partial \xi$ are defined for all $\xi$ not belonging to the null set $N_{\xi}$, and for all $\eta$ larger than $-\xi$; analogously, the functions $\partial \tilde{u}^{r} / \partial \eta$ and $\partial \tilde{u}^{l} / \partial \eta$ are defined for all $\eta$ not belonging to the null set $N_{\eta}$ and for all $\xi$ larger than $-\eta$.

[5, Prop. V.2 and Cor. V.4] tell us that

$$
\begin{aligned}
& \frac{\partial u^{r}}{\partial \xi}(\cdot, \sigma(\cdot)) \in L_{\mathrm{loc}}^{2}\left(\mathbb{R} ;\left(1+\sigma^{\prime}\right) d x\right), \\
& \frac{\partial u^{r}}{\partial \eta}(\cdot, \sigma(\cdot)) \in L_{\mathrm{loc}}^{2}\left(\mathbb{R} ;\left(1-\sigma^{\prime}\right) d x\right), \\
& \frac{\partial u^{l}}{\partial \xi}(\cdot, \sigma(\cdot)) \in L_{\mathrm{loc}}^{2}\left(\{x: \sigma(x)>0\},\left(1+\sigma^{\prime}\right) d x\right), \\
& \frac{\partial u^{l}}{\partial \eta}(\cdot, \sigma(\cdot)) \in L_{\mathrm{loc}}^{2}\left(\{x: \sigma(x)>0\},\left(1-\sigma^{\prime}\right) d x\right) .
\end{aligned}
$$

Note that the above traces are not continuous functions of $u$. We have the following example:

$$
u_{n}(x, t)= \begin{cases}1+\frac{1}{n}-t & \text { if } t \leq 1+\frac{1}{n} \\ t-\left(1+\frac{1}{n}\right) & \text { if } t \geq 1+\frac{1}{n}\end{cases}
$$

Then

$$
\frac{\partial u^{r}}{\partial \xi}(x, 1)=\frac{1}{\sqrt{2}} \quad \forall x, \quad \frac{\partial u_{n}^{r}}{\partial \xi}(x, 1)=-\frac{1}{\sqrt{2}} \quad \forall x, \quad \forall n
$$


We may now state the following result of weak convergence of the penalization:

THEOREM 13. Given initial conditions $u_{0} \in L_{\mathrm{loc}}^{1}(\mathbb{R})$ and $u_{1} \in L_{\mathrm{loc}}^{2}(\mathbb{R})$ such that $u_{0} \geq \varphi$ and $u_{1} \geq 0$ almost everywhere on the set $\left\{x: u_{0}(x)=\varphi(x)\right\}$, there exists a function $u$ such that

$$
\begin{gathered}
u \in V, \\
u \geq \varphi, \\
\square u \geq 0, \\
\operatorname{supp} \square u \subset\{(x, t): u(x, t)=\varphi(x)\}, \\
\int_{a}^{b}\left[\left|\frac{\partial u^{r}}{\partial \xi}(x, \sigma(x))\right|^{2}\left(1+\sigma^{\prime}(x)\right)+\left|\frac{\partial u^{r}}{\partial \eta}(x, \sigma(x))\right|^{2}\left(1-\sigma^{\prime}(x)\right)\right] d x \\
\int_{a}^{b}\left[\left|\frac{\partial u^{l}}{\partial \xi}(x, \sigma(x))\right|^{b}\left(\left|u_{1}\right|^{2}+\left|\frac{d u_{0}}{\partial x}\right|^{2}\right) d x,\right. \\
\leq \int_{b}^{a}\left(\left|u_{1}\right|^{2}+\left|\frac{d u_{0}}{d x}\right|^{2}\right) d x,
\end{gathered}
$$

for all Lipschitz continuous functions $\sigma$, with Lipschitz constant 1 , such that $\sigma(a)=\sigma(b)=0$, $\sigma>0$ on $(a, b)$,

$$
\begin{gathered}
u(x, 0)=u_{0}(x) \\
\frac{\partial u}{\partial t}(x, 0)=u_{1}(x) \quad \text { if } u_{0}(x)>\varphi(x) \\
\left|\frac{\partial u}{\partial t}(x, 0)\right| \leq u_{1}(x) \quad \text { if } u_{0}(x)=\varphi(x) .
\end{gathered}
$$

Proof. From estimates (123) and (124), we can see that we can extract a subsequence $u_{\mu}$ such that

$$
u_{\mu} \rightarrow u \quad \text { weakly } * \text { in } V \text {. }
$$

The weak $*$ topology on $V$ is defined by the semi-norms

$$
\left|\int u f_{1}\right|+\left|\int u_{x} f_{2}\right|+\left|\int u_{t} f_{3}\right|
$$

where $f_{1}, f_{2}$ and $f_{3}$ are in $L^{1}\left(\mathbb{R}^{+} ; L^{2}(\mathbb{R})\right)$ with compact support in $\mathbb{R} \times[0, \infty)$. We deduce from (133) that

$$
u_{\mu} \rightarrow u \text { in } C^{0}\left(\mathbb{R} \times \mathbb{R}^{+}\right) \quad \text { with the compact topology. }
$$

Possibly with a new extraction

(135) $\frac{1}{\mu}\left(u_{\mu}-\varphi\right) \rightarrow \nu \quad$ weakly in $M\left(\mathbb{R} \times \mathbb{R}^{+}\right)$the set of measures on $\mathbb{R} \times \mathbb{R}^{+}$.

Therefore

$$
\square u=\nu \geq 0
$$


Relation (123) gives a bound on $\left(\left(u_{\mu}-\varphi\right)^{-}\right)^{2} / \mu$ in $L_{\mathrm{loc}}^{1}$ and thus

$$
u \geq \varphi \text {. }
$$

To check (129), let $\left(x_{0}, t_{0}\right)$ be a point such that $u\left(x_{0}, t_{0}\right)>\varphi\left(x_{0}\right)$; thanks to (134) we can find a neighborhood $U$ of $\left(x_{0}, t_{0}\right)$ and a $\mu_{0}$ such that $u_{\mu}(x, t)-\varphi(x)$ $\geq \frac{1}{4}\left(u\left(x_{0}, t_{0}\right)-\varphi\left(x_{0}\right)\right) \forall \mu<\mu_{0}$, for all $(x, t) \in U$.

Therefore

$$
\left.\square u_{\mu}\right|_{U}=0 \text { for } \mu<\mu_{0},
$$

and in the limit $\left.\square u\right|_{U}=0$. This proves (129).

To prove (130), let $\sigma$ be given, and $\varepsilon_{0}$ be a positive number. Let us define for $|\varepsilon|<\varepsilon_{0}$

$$
\sigma_{\varepsilon}(x)= \begin{cases}\sigma(x)+\varepsilon & \text { if } x \in\left[a+\varepsilon_{0}, b-\varepsilon_{0}\right], \\ x-a+\varepsilon-\varepsilon_{0} & \text { if } x \in\left[a+\varepsilon_{0}-\varepsilon, a+\varepsilon_{0}\right] \\ -x+b-\varepsilon_{0}+\varepsilon & \text { if } x \in\left[b-\varepsilon_{0}, b+\varepsilon-\varepsilon_{0}\right] .\end{cases}
$$

Then (123) implies

$$
\begin{array}{r}
\int_{-\varepsilon^{\prime}}^{\varepsilon^{\prime \prime}} d \varepsilon \int_{a+\varepsilon_{0}}^{b-\varepsilon_{0}}\left[\left|\frac{\partial u_{\mu}}{\partial \xi}\left(x, \sigma_{\varepsilon}(x)\right)\right|^{2}\left(1+\sigma_{\varepsilon}^{\prime}(x)\right)\right. \\
\left.+\left|\frac{\partial u_{\mu}}{\partial \eta}\left(x, \sigma_{\varepsilon}(x)\right)\right|^{2}\left(1-\sigma_{\varepsilon}^{\prime}(x)\right)\right] d x \\
\leq\left(\varepsilon^{\prime}+\varepsilon^{\prime \prime}\right) \int_{a-\varepsilon_{0}}^{b+\varepsilon_{0}}\left(\left|\frac{d u_{0}}{d x}\right|^{2}+\left|u_{1}\right|^{2}\right) d x .
\end{array}
$$

But the left-hand side term of (138) can be written as

$$
\int_{a+\varepsilon_{0}}^{b-\varepsilon_{0}} d x \int_{\sigma(x)-\varepsilon^{\prime}}^{\sigma(x)+\varepsilon^{\prime \prime}}\left\{\left|\frac{\partial u_{\mu}}{\partial \xi}(x, t)\right|^{2}\left(1+\sigma^{\prime}(x)\right)+\left|\frac{\partial u_{\mu}}{\partial \eta}\right|^{2}(x, t)\left(1-\sigma^{\prime}(x)\right)\right\} d t
$$

and we can take a weak limit in this double integral, thanks to (133).

Thus we can rewrite (138) without the index $\mu$ :

$$
\begin{gathered}
\int_{-\varepsilon^{\prime}}^{\varepsilon^{\prime \prime}} d \varepsilon \int_{a+\varepsilon_{0}}^{b-\varepsilon_{0}}\left[\left|\frac{\partial u}{\partial \xi}\left(x, \sigma_{\varepsilon}(x)\right)\right|^{2}\left(1+\sigma_{\varepsilon}^{\prime}(x)\right)+\left|\frac{\partial u}{\partial \eta}\left(x, \sigma_{\varepsilon}(x)\right)\right|^{2}\left(1-\sigma_{\varepsilon}^{\prime}(x)\right)\right] d x \\
\leq\left(\varepsilon^{\prime}+\varepsilon^{\prime \prime}\right) \int_{a-\varepsilon_{0}}^{b+\varepsilon_{0}}\left(\left|\frac{d u_{0}}{d x}\right|^{2}+\left|u_{1}\right|^{2}\right) d x .
\end{gathered}
$$

Taking $\varepsilon^{\prime}=0$ in (139) and letting $\varepsilon^{\prime \prime}$ tend to zero, we obtain

$$
\begin{gathered}
\int_{a+\varepsilon_{0}}^{b-\varepsilon_{0}}\left[\left|\frac{\partial u^{r}}{\partial \xi}(x, \sigma(x))\right|^{2}\left(1+\sigma^{\prime}(x)\right)+\left|\frac{\partial u^{r}}{\partial \eta}(x, \sigma(x))\right|^{2}\left(1-\sigma^{\prime}(x)\right)\right] d x \\
\leq \int_{a-\varepsilon_{0}}^{b+\varepsilon_{0}}\left(\left|\frac{d u_{0}}{d x}\right|^{2}+\left|u_{1}\right|^{2}\right) d x .
\end{gathered}
$$

Letting $\varepsilon_{0}$ go to zero, we obtain the first relation of (130). If we take $\varepsilon^{\prime \prime}=0$ and let $\varepsilon^{\prime}$ and then $\varepsilon_{0}$ tend to zero, we obtain the second relation of (130). 
The initial condition (131) is obviously satisfied. It remains to check (132). For this purpose, let us take, in (137), $\sigma(x)=0$ on $[a, b]$. Then, ultimately we get

$$
\int_{a}^{b}\left[\left|\frac{\partial u^{r}}{\partial \xi}(x, 0)\right|^{2}+\left|\frac{\partial u^{r}}{\partial \eta}(x, 0)\right|^{2}\right] d x \leq \int_{a}^{b}\left(\left|\frac{d u_{0}}{d x}\right|^{2}+\left|u_{1}\right|^{2}\right) d x .
$$

Using the identity

$$
\left|\frac{\partial u^{r}}{\partial \xi}(x, 0)\right|^{2}+\left|\frac{\partial u^{r}}{\partial \eta}(x, 0)\right|^{2}=\left|\frac{d u_{0}}{d x}\right|^{2}+\left|\frac{\partial u}{\partial t}(x, 0+0)\right|^{2}
$$

which takes into account (131), we have

$$
\int_{b}^{a}\left|\frac{\partial u}{\partial t}(x, 0+0)\right|^{2} d x \leq \int_{a}^{b}\left|u_{1}\right|^{2} d x
$$

As $a$ and $b$ are arbitrary, we have eventually

$$
\left|\frac{\partial u}{\partial t}(x, 0+0)\right| \leq\left|u_{1}(x)\right| \text { a.e. on } \mathbb{R} .
$$

When $u_{0}(x)>\varphi(x)$, we have the first part of (132), as locally, $\nu=\square u=0$.

We shall now study the relation between the strong convergence of $\partial u_{\lambda} / \partial x$ and $\partial u_{\lambda} / \partial t$, and the verification of the energy condition (11).

LEMMA 14. Let $u_{\lambda}$ be a sequence of solutions of (122), converging weakly* to a solution $u$ of (126) $-(132)$. Then, $u$ satisfies the energy condition (11) if and only if $\partial u_{\lambda} / \partial t$ and $\partial u_{\lambda} / \partial x$ converge to $\partial u / \partial t$ and $\partial u / \partial x$ respectively, strongly in $L_{\text {loc }}^{2}(\mathbb{R} \times[0, \infty))$.

Proof. Notice first that as $\left(\left(u_{\mu}-\varphi\right)^{-} / \mu\right) \cdot 1_{K}$ converges to $\nu \cdot 1_{K}$ in $M\left(\mathbb{R} \times \mathbb{R}^{+}\right)$ weakly, for all compact $K$, and as $\left(u_{\mu}-\varphi\right)^{-}$converges to zero uniformly on compact sets, then

$$
\int_{K}\left[\frac{\left(\left(u_{\mu}-\varphi\right)^{-}\right)^{2}}{\mu}\right] d x^{\prime} d t^{\prime} \rightarrow 0
$$

for any compact set $K$.

Let $\sigma_{h}\left(x^{\prime}\right)=h-\left|x-x^{\prime}\right|$. Then we have the identity, for any function $v$,

$$
\begin{aligned}
& \int_{0}^{t} d h \int_{x-h}^{x+h}\left[\left|\frac{\partial v}{\partial \xi}\left(x, \sigma_{h}(x)\right)\right|^{2}\left(1+\sigma_{h}^{\prime}(x)\right)+\left|\frac{\partial v}{\partial \eta}\left(x, \sigma_{h}(x)\right)\right|^{2}\left(1-\sigma_{h}^{\prime}(x)\right)\right] d x \\
& =\int_{x-t}^{x} d x^{\prime} \int_{0}^{t+x-x^{\prime}} 2\left|\frac{\partial v}{\partial \xi}\left(x^{\prime}, t^{\prime}\right)\right|^{2} d t^{\prime}+\int_{x}^{x+t} d x^{\prime} \int_{0}^{t-x+x^{\prime}} 2\left|\frac{\partial v}{\partial \eta}\left(x^{\prime}, t^{\prime}\right)\right|^{2} d t^{\prime}
\end{aligned}
$$

If the limit of the sequence $u_{\mu}$ satisfies (11), then the value of (141) for $v=u$ is

$$
\int_{0}^{t} d h \int_{x-h}^{x+h}\left(\left|\frac{d u_{0}}{d x}\right|^{2}+\left|u_{1}\right|^{2}\right) d x
$$

The value of (141) for $v=u_{\mu}$ is

$$
\int_{0}^{t} d h \int_{x-h}^{x+h}\left(\left|\frac{d u_{0}}{d x}\right|^{2}+\left|u_{1}\right|^{2}\right) d x-\int_{0 \leq t^{\prime} \leq t-\left|x-x^{\prime}\right|} \frac{1}{\mu}\left(\left(u_{\mu}-\varphi\right)^{-}\right)^{2} d x^{\prime} d t
$$


And according to (140), the limit of (143) is (142). Therefore, as $\partial u_{\mu} / \partial \xi$ (resp. $\partial u_{\mu} / \partial \eta$ ) converges weakly to $\partial u / \partial \xi($ resp. $\partial u / \partial \eta)$ in $L_{\text {loc }}^{2}\left([0, \infty) \times \mathbb{B}^{+}\right)$, and

$$
\lim _{\mu \rightarrow 0} \int_{A_{x, t}}\left|\frac{\partial u_{\mu}}{\partial \xi}\right|^{2} d x^{\prime} d t^{\prime}+\int_{B_{x, t}}\left|\frac{\partial u_{\mu}}{\partial \eta}\right|^{2} d x^{\prime} d t^{\prime}=\int_{A_{x, t}}\left|\frac{\partial u}{\partial \xi}\right|^{2} d x^{\prime} d t^{\prime}+\int_{B_{x, t}}\left|\frac{\partial u}{\partial \eta}\right|^{2} d x^{\prime} d t^{\prime}
$$

where $A_{x, t}=\left\{\left(x^{\prime}, t^{\prime}\right) \in T_{x, t}^{-} / x^{\prime} \leq 0\right\}, B_{x, t}=T_{x, t}^{-} \backslash A_{x, t}$, we can conclude that the convergence of $\partial u_{\mu} / \partial \xi$ and $\partial u_{\mu} / \partial \eta$ to $\partial u / \partial \xi$ and $\partial u / \partial \eta$ is strong.

Conversely, if $\partial u_{\mu} / \partial \xi$ (resp. $\partial u_{\mu} / \partial \eta$ ) converges strongly to $\partial u / \partial \xi$ (resp. $\partial u / \partial \eta$ ), then it is straightforward to pass to the limit in (11).

5.2. Strong convergence when the obstacle is zero and the initial characteristic derivatives are of bounded variation. The first step in this study is to notice that if $w$ is an affine function, then the penalized solution converges to the solution of $\left(P_{\infty}\right)$ which conserves the energy.

LEMMA 15. Let there be given initial conditions

$$
\begin{aligned}
& u(x, 0)=a-b x \geq 0 \quad \text { on }\left[x_{0}-t_{0}, x_{0}+t_{0}\right], \\
& u_{t}(b, 0)=-c<0,
\end{aligned}
$$

and suppose that the free solution $w(x, t)=a-b x-c t$ is such that

$$
w\left(x_{0}, t_{0}\right)<0 \text {. }
$$

Then the solution $u_{\lambda}$ of (122) with initial conditions (144) is given by

$$
u_{\lambda}(x, t)= \begin{cases}a-b x-c t & \text { for } b x+c t<a, \\ \sqrt{\lambda\left(c^{2}-b^{2}\right)} \sin \frac{c t+b x-a}{\sqrt{\lambda\left(c^{2}-b^{2}\right)}} & \text { for } a \leq b x+c t \leq a+\pi \sqrt{\lambda\left(c^{2}-b^{2}\right)}, \\ b x+c t-a-\pi \sqrt{\lambda\left(c^{2}-b^{2}\right)} & \text { for } b x+c t \geq a+\pi \sqrt{\lambda\left(c^{2}-b^{2}\right)} .\end{cases}
$$

Therefore $u_{\lambda}$ converges strongly in $H^{1}\left(T_{x_{0}, t_{0}}^{-}\right)$to the solution of $\left(P_{x_{0}, t_{0}}\right)$.

Proof. Let us compute the solution of $\left(P_{x_{0}, t_{0}}\right)$ :

$$
E=\left\{(x, t) \in T_{x_{0}, t_{0}}^{-}: a-b x-c t<0\right\} .
$$

We see at once that the slope of the line $a=b x+c t$ is smaller than 1 , in absolute value. Therefore $I=E$, and

$$
u(x, t)= \begin{cases}a-b x-c t & \text { if } a-b x-c t \geq 0, \\ b x+c t-a & \text { if } a-b x-c t \leq 0 .\end{cases}
$$

Let us look for the solution of (122) with initial conditions (144) under the form

$$
u_{\lambda}(x, t)=f_{\lambda}(b x+c t) \text {. }
$$

Then $f_{\lambda}$ must satisfy the ordinary differential equation

$$
\left(c^{2}-b^{2}\right) f^{\prime \prime}-\frac{1}{\lambda} f^{-}=0
$$

with the initial conditions

$$
f_{\lambda}(a)=0, \quad f_{\lambda}^{\prime}(a)=-1 .
$$

This problem can be solved immediately and gives (145). Clearly the limit of the sequence $u_{\lambda}$ is $u$, and Lemma 14 allows us to conclude the proof. 
Remark 16. Suppose we replace the function $r^{-}$by a function $\psi$ such that

$$
\begin{aligned}
& \psi(x)=0 \quad \text { if } x \geq 0 \\
& \psi(x)>0 \text { if } x<0, \\
& \psi \text { is continuous, strictly decreasing on }(-\infty, 0), \\
& \psi(-\infty)=\infty
\end{aligned}
$$

Then the penalized problem

$$
\begin{aligned}
& \square \hat{u}_{\lambda}-\frac{1}{\lambda} \psi\left(\hat{u}_{\lambda}-\varphi\right)=0, \\
& \hat{u}_{\lambda}(x, 0)=u_{0}(x), \\
& \frac{\partial \hat{u}_{\lambda}}{\partial t}(x, 0)=u_{1}(x)
\end{aligned}
$$

can be studied as above; we get Theorem 14 with almost no change in the proof. Moreover, a phase plane analysis shows easily that in the case of initial data (144) the limit of $u_{\lambda}$ is the function (146). We chose the specific penalization (122) because of its simplicity. We need an integral solution of the linear Klein-Gordon equation with initial values given on a curve $t=\sigma(x)$. This is the object of the next lemma.

LEMMA 17. Let $w$ be a solution of the wave equation on the set $S=\{(x, t) / \sigma(x) \leq t \leq$ $\left.t_{0}-\left|x-x_{0}\right|\right\}$ where $\sigma$ is a Lipschitz continuous function with Lipschitz constant 1.

Then the unique solution on $S$ of the problem

$$
\begin{aligned}
& \square u+\frac{1}{\lambda} u=0, \\
& u(x, \sigma(x))=w(x, \sigma(x)), \\
& \frac{\partial u}{\partial t}(x, \sigma(x))=\frac{\partial w}{\partial t}(x, \sigma(x)) \quad \text { a.e.on }\left\{x:\left|\sigma^{\prime}(x)\right|<1\right\}
\end{aligned}
$$

is given by

$$
u(x, t)=w(x, t)-\frac{1}{2} \int_{S \cap T_{x, t}} g_{0}\left(\frac{\sqrt{\left(t-t^{\prime}\right)^{2}-\left(x-x^{\prime}\right)^{2}}}{\sqrt{\lambda}}\right) w\left(x^{\prime}, t^{\prime}\right) d x^{\prime} d t^{\prime}
$$

where

$$
g_{0}(y)=\sum_{n \geq 0} \frac{(-1)^{n}}{n !}\left(\frac{y}{2}\right)^{2 n}
$$

is the Bessel function $\mathscr{G}_{0}$.

Proof. We verify that if $w$ is a solution of the wave equation in the whole plane and if

$$
\bar{w}(x, t)= \begin{cases}0 & \text { if } t<\sigma(x) \\ w(x, t) & \text { if } t \geq \sigma(x)\end{cases}
$$

then

$$
\begin{aligned}
\langle\square \bar{w}, \varphi\rangle=- & \int w(x, \sigma(x))\left(\varphi_{t}(x, \sigma(x))+\sigma^{\prime}(x) \varphi_{x}(x, \sigma(x))\right) d x \\
& +\int\left[w_{t}(x, \sigma(x))+\sigma^{\prime}(x) w_{x}(x, \sigma(x))\right] \varphi(x, \sigma(x)) d x .
\end{aligned}
$$


Solving (147) amounts to finding a solution of

$$
\begin{aligned}
& \left.\left(\square u+\frac{1}{\lambda} u\right)\right|_{\{(x, t): t>\sigma(x)\}}=0, \\
& \left.u(x, t)\right|_{\{(x, t): t \leq \sigma(x)\}}=0, \\
& u(x, \sigma(x))=w(x, \sigma(x)), \\
& \frac{\partial u}{\partial t}(x, \sigma(x))=\frac{\partial w}{\partial t}(x, \sigma(x)) \quad \text { a.e. on }\left\{x:\left|\sigma^{\prime}(x)\right|<1\right\},
\end{aligned}
$$

which can be written as

$$
u=\bar{w}-\frac{1}{\lambda} \mathcal{E} * u
$$

where $\mathscr{E}$ is the elementary solution of the wave equation defined by

$$
\mathscr{E}(x, t)= \begin{cases}\frac{1}{2} & \text { if } t \geq x \\ 0 & \text { elsewhere }\end{cases}
$$

The convolution equation (150) has a unique solution given by

$$
u=\sum_{n=0}^{\infty} \frac{(-1)^{n}}{\lambda^{n}}\left(\begin{array}{c}
n \\
* \\
k=1
\end{array}\right) * \bar{w} .
$$

By a simple inductive calculation in characteristic coordinates, we obtain:

$$
\left(\begin{array}{c}
\underset{k}{*} \tilde{\delta} \\
k=1
\end{array}\right)(\xi, \eta)=\tilde{\mathscr{E}} \cdot\left(\frac{\xi \eta}{2}\right)^{n-1} \frac{1}{((n-1) !)^{2}} .
$$

Therefore

$$
\sum_{n=1}^{\infty} \frac{(-1)^{n}}{\lambda^{n}}(\underset{k=1}{*} \mathcal{E})(x, t)=-\frac{1}{2 \lambda} \mathcal{E} \cdot g_{0}\left(\frac{\sqrt{t^{2}-x^{2}}}{\lambda}\right) .
$$

Together with (153), formula (151) gives (149).

We can now state the theorem of convergence for penalized solutions:

THEOREM 18. Let $u_{0}$ and $u_{1}$ be such that

$$
\frac{d u_{0}}{d x} \text { and } u_{1} \text { are locally of bounded variation }
$$

and suppose that they satisfy the compatibility condition (122). Then the solution $u_{\lambda}$ of (122) converges to the solution of $\left(\mathrm{P}_{\infty}\right)$ when $\lambda$ goes to zero.

Proof. Let us first notice that on $I^{c}$, the complement of the domain of influence, we have, if $u$ is the solution of $(\mathrm{P})$ :

Therefore

$$
\square u=0, \quad u \geq 0 \quad \text { on } I^{c} \text {. }
$$

$$
u_{\lambda}=u \text { on } \overline{I^{c}}
$$


and, in particular,

$$
\begin{aligned}
& u_{\lambda}(x, \tau(x))=u(x, \tau(x))=w(x, \tau(x)), \\
& \frac{\partial u_{\lambda}}{\partial t}(x, \tau(x))=\frac{\partial u}{\partial t}(x, \tau(x)-0)=\frac{\partial w}{\partial t}(x, \tau(x)) \text { a.e. on }\left\{x:\left|\tau^{\prime}(x)\right|<1\right\},
\end{aligned}
$$

where we recall that $\tau$, the line of influence, is Lipschitz continuous, with Lipschitz constant 1 .

We shall now use assumption (154) to obtain more information about the line of influence. We need the following notation (see Fig. 3):

$$
\begin{aligned}
& Q_{1}=\{(x, t): x \geq|t|\}, \\
& Q_{2}=\{(x, t): t \leq|x|\}, \\
& Q_{3}=\{(x, t): x \leq-|t|\}, \\
& Q_{4}=\{(x, t): t \leq-|x|\} .
\end{aligned}
$$

We shall denote

$$
\frac{\partial w}{\partial t}\left(x, t: Q_{i}\right)=\lim _{\substack{(h, k) \rightarrow 0 \\(h, k) \in Q_{i}}} \frac{\partial w}{\partial t}(x+h, t+k) .
$$

Thanks to (154), $(\partial w / \partial t)\left(x, t ; Q_{i}\right)$ is defined for $1 \leq i \leq 4$, and we have the formula

$$
\frac{\partial w}{\partial t}\left(x, t ; Q_{1}\right)=\frac{1}{\sqrt{2}}\left[\frac{\partial w^{l}}{\partial \xi}(x, t)+\frac{\partial w^{r}}{\partial \eta}(x, t)\right],
$$

with notation as in (125). We have analogous formulae for the three other limits.

LEMMA 19. Let $x$ be such that $\tau^{\prime}(x)$ is defined and $\left|\tau^{\prime}(x)\right|<1$. Suppose that

$$
\max _{1 \leq i \leq 4} \frac{\partial w}{\partial t}\left(x, t ; Q_{i}\right)<0 .
$$

Then there exists a neighborhood $(x-\varepsilon, x+\varepsilon)$ of $x$ such that $\left|x^{\prime}-x\right|<\varepsilon \Rightarrow \tau^{\prime}\left(x^{\prime}\right)$ has left and right limits at every point and $\left|\tau^{\prime}\left(x^{\prime} \pm 0\right)\right|<1$; moreover

$$
\sup _{1 \leq i \leq 4} \frac{\partial w}{\partial t}\left(x^{\prime}, \tau\left(x^{\prime}\right) ; Q_{i}\right) \leq-l<0 .
$$

Proof. The hypothesis (159) implies that, in a neighborhood $N$ of $(x, \tau(x))$

$$
\sup _{1<i<4} \frac{\partial w}{\partial t}\left(x^{\prime}, t^{\prime} ; Q_{i}\right) \leq-l<0 ;
$$

therefore $w\left(x^{\prime}, \cdot\right)$ is strictly decreasing for $x^{\prime}$ close enough to $x$, and moreover, if $k$ is so chosen that $w_{t}(x, \tau(x))+k w_{x}(x, \tau(x))<0$, then

$$
w(x+k h, \tau(x)+h)<0 .
$$

Thus, there exists a unique solution to the problem

$$
\begin{aligned}
& w\left(x^{\prime}, \sigma\left(x^{\prime}\right)\right)=0, \\
& \max \left(\left|x-x^{\prime}\right|,\left|\sigma\left(x^{\prime}\right)-\tau(x)\right|\right) \leq \alpha, \quad \text { where } \alpha \text { is a small positive number. }
\end{aligned}
$$




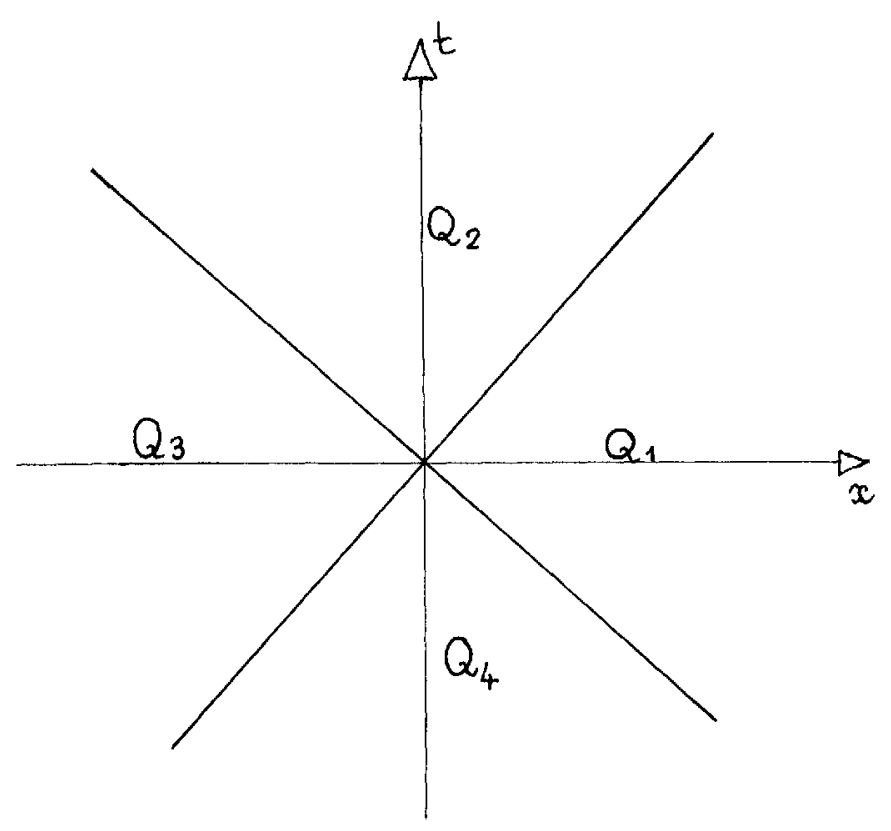

FIG. 3. The regions $Q_{i}, i=1, \cdots, 4$ of the $(x, t)$-plane.

To prove that $\sigma$ is identical to $\tau$ in an interval $\left[x-\alpha^{\prime}, x+\alpha^{\prime}\right]$ where $\alpha^{\prime}$ may be smaller than $\alpha$, we have to check that

$$
\left|\sigma^{\prime}\left(x^{\prime}\right)\right|<1 \quad \text { a.e. on }\left[x-\alpha^{\prime}, x+\alpha^{\prime}\right] .
$$

The function $\sigma$ is continuous indeed, as $w$ is continuous and $t^{\prime}=\sigma\left(x^{\prime}\right)$ is the unique solution of $w\left(x^{\prime}, t^{\prime}\right)=0$ in $N$. We may not directly differentiate the relation $w\left(x^{\prime}, \sigma\left(x^{\prime}\right)\right)$ $=0$, as we do not have the assumptions of the implicit function theorem. But, with the very same argument as in this theorem, and using notation (157) and its analogue for $\partial u / \partial x$, we have

$$
\begin{aligned}
w\left(x^{\prime}+h, \sigma(x+h)\right)=w & \left(x, \sigma\left(x^{\prime}\right)\right)+w_{x}\left(x^{\prime}, \sigma\left(x^{\prime}\right) ; Q_{1}\right) h \\
& +w_{t}\left(x^{\prime}, \sigma\left(x^{\prime}\right) ; Q_{1}\right)\left(\sigma\left(x^{\prime}+h\right)-\sigma\left(x^{\prime}\right)\right) \\
& +\varepsilon_{1}\left(|h|+\left|\sigma\left(x^{\prime}+h\right)-\sigma\left(x^{\prime}\right)\right|\right)
\end{aligned}
$$

for all $h$ such that $\left(h, \sigma\left(x^{\prime}+h\right)-\sigma\left(x^{\prime}\right)\right) \in Q_{1}$.

Here $\varepsilon_{1}$ is a function such that

$$
\lim _{k \rightarrow 0} \frac{\varepsilon_{1}(k)}{k}=0
$$

By a standard argument

$$
\lim _{\left(h, \sigma\left(x^{\prime}+h\right)-\sigma\left(x^{\prime}\right)\right) \in Q_{1}}\left[\frac{\sigma\left(x^{\prime}+h\right)-\sigma\left(x^{\prime}\right)}{h}\right]=-\frac{w_{x}\left(x^{\prime}, \sigma\left(x^{\prime}\right) ; Q_{1}\right)}{w_{t}\left(x^{\prime}, \sigma\left(x^{\prime}\right) ; Q_{1}\right)} .
$$

The same result holds in the three other quadrants $Q_{2}, Q_{3}$ and $Q_{4}$, and by choosing $\alpha^{\prime}$ adequately small we shall have

$$
\left|\frac{w_{x}\left(x^{\prime}, \sigma\left(x^{\prime}\right) ; Q_{i}\right)}{w_{t}\left(x^{\prime}, \sigma\left(x^{\prime}\right) ; Q_{i}\right)}\right| \leq 1-\varepsilon \text { for }\left|x-x^{\prime}\right| \leq \alpha^{\prime},
$$


and thus

$$
\begin{gathered}
\left(h, \sigma\left(x^{\prime}+h\right)-\sigma\left(x^{\prime}\right)\right) \in Q_{1} \quad \text { for } h>0 \text { small enough, } \\
\left(h, \sigma\left(x^{\prime}+h\right)-\sigma\left(x^{\prime}\right)\right) \in Q_{3} \quad \text { for } h<0,|h| \text { small enough, } \\
\left|\sigma^{\prime}\left(x^{\prime}\right)\right| \leq 1-\varepsilon \quad \text { a.e. on }\left[x-\alpha^{\prime}, x+\alpha^{\prime}\right],
\end{gathered}
$$

$\tau\left(x^{\prime}\right)=\sigma\left(x^{\prime}\right)$, and $\tau^{\prime}$ has right and left limits at all points of $\left[x-\alpha^{\prime}, \mathrm{x}+\alpha^{\prime}\right]$.

Let us compare locally the solution of the linear Klein-Gordon equation (147) to the solution of an approaching problem with simpler initial data. Let

$$
\begin{aligned}
& \tau\left(x_{0}\right)=t_{0}, \quad \tau_{0}^{\prime}\left(x_{0}\right)=m=-\frac{w_{x}\left(x_{0}, t_{0}\right)}{w_{t}\left(x_{0}, t_{0}\right)}, \\
& \tau_{0}(x)=t_{0}+m\left(x-x_{0}\right), \\
& w_{0}(x, t)=w_{t}\left(x_{0}, t_{0}\right)\left(t-t_{0}\right)+w_{x}\left(x_{0}, t_{0}\right)\left(x-x_{0}\right), \\
& u_{0}\left(x, \tau_{0}(x)\right)=w_{0}\left(x, \tau_{0}(x)\right)=0, \\
& \frac{\partial u_{0}}{\partial t}\left(x, \tau_{0}(x)\right)=\frac{\partial w_{0}}{\partial t}\left(x, \tau_{0}(x)\right)=w_{t}\left(x_{0}, t_{0}\right), \\
& S_{0}=\left\{(x, t): t>\tau_{0}(x)\right\} .
\end{aligned}
$$

Then:

$$
u_{0}(x, t)=\sqrt{\lambda\left(1-m^{2}\right)} w_{t}\left(x_{0}, t_{0}\right) \sin \frac{t-t_{0}-m\left(x-x_{0}\right)}{\sqrt{\lambda\left(1-m^{2}\right)}} .
$$

With the help of (148), we have

$$
\begin{gathered}
u_{\lambda}(x, t)-u_{0}(x, t)=w(x, t)-w_{0}(x, t) \\
-\frac{1}{2 \lambda} \int_{T_{x, t}^{\prime}} g_{0}\left(\frac{\sqrt{\left(t-t^{\prime}\right)^{2}-\left(x-x^{\prime}\right)^{2}}}{\lambda}\right) \\
\cdot\left[\left(w \cdot 1_{S}\right)\left(x^{\prime}, t^{\prime}\right)-\left(w_{0} \cdot 1_{s_{0}}\right)\left(x^{\prime}, t^{\prime}\right)\right] d x^{\prime} d t^{\prime} .
\end{gathered}
$$

Let us estimate (163) for $x$ and $t$ such that

$$
\left|x-x_{0}\right|+\left|t-t_{0}\right| \leq C \sqrt{\lambda},
$$

and under the hypotheses that $\left|\tau^{\prime}\left(x_{0}\right)\right|<1$ and that $w_{t}\left(x_{0}, t_{0}\right)$ and $w_{x}\left(x_{0}, t_{0}\right)$ are well defined. Then

$$
\left|w(x, t)-w_{0}(x, t)\right| \leq o\left(\left|x-x_{0}\right|+\left|t-t_{0}\right|\right)=o(\sqrt{\lambda}) .
$$

To estimate the integral, let us first note that

$$
\left|w \cdot 1_{S}-w_{0} \cdot 1_{S_{0}}\right| \leq\left|w-w_{0}\right| \cdot 1_{S \cup S_{0}} .
$$

This relation comes from the fact that, locally, $w \cdot 1_{S^{\prime}}=-w^{-}$and $w_{0} \cdot 1_{S_{0}}=-w_{0}^{-}$. We define new variables $X$ and $T$ by

$$
t-t^{\prime}=T \sqrt{\lambda}, \quad x-x^{\prime}=X \sqrt{\lambda} .
$$


Then the integral expression in (163) is estimated by

$$
\begin{aligned}
& \lambda \int_{T_{0,0}^{+}}\left|g_{0}\left(\sqrt{T^{2}-X^{2}}\right)\right| \mid w(x-X \sqrt{\lambda}, t-T \sqrt{\lambda})- w_{0}(x-X \sqrt{\lambda}, t-T \sqrt{\lambda}) \mid \\
& \cdot 1_{S \cup s_{0}}(x-X \sqrt{\lambda}, t-T \sqrt{\lambda}) d X d T .
\end{aligned}
$$

But

$$
\left|w(x-X \sqrt{\lambda}, t-T \sqrt{\lambda})-w_{0}(x-X \sqrt{\lambda}, t-T \sqrt{\lambda})\right| \leq o\left(\left|x-X \sqrt{\lambda}-x_{0}\right|+\left|t-T \sqrt{\lambda}-t_{0}\right|\right),
$$

and we have to check that $\left\{(X, T) \in T_{00}^{+}:(x-X \sqrt{\lambda}, t-T \sqrt{\lambda}) \in S \cup S_{0}\right\}$ is bounded. This set can be written as

$$
\left\{(X, T):|X| \leq T \leq \frac{t}{\sqrt{\lambda}}-\min \left(\frac{\tau(x-X \sqrt{\lambda}), \tau_{0}(x-X \sqrt{\lambda})}{\sqrt{\lambda}}\right)\right\},
$$

and using the fact that $\left|\tau^{\prime}\left(x_{0}\right)\right|<1$, this set is bounded under the condition (164).

Thus, immediately,

$$
\left|u(x, t)-u_{0}(x, t)\right|=o(\sqrt{\lambda}) .
$$

A consequence of (165) is that, for $\lambda$ sufficiently small, the solution $u$ of (147) is negative on the set

$$
T_{x_{0}, t_{0}+(\pi-\varepsilon)}^{-} \sqrt{\lambda\left(1-m^{2}\right)} \cap\{(x, t): t \geq \tau(x)\} .
$$

This uses the fact that $u_{t}<0$ on a neighborhood of $x_{0}$, as was proved in Lemma 19.

Therefore, on the set (166), the solution of the penalized problem (122) is the solution of the linear problem (147), for $\lambda$ small enough. We have thus, for $(x, t)$ on the set (166):

$$
\begin{aligned}
\frac{\partial u_{\lambda}}{\partial t}\left(x^{\prime}, t^{\prime}\right)=\frac{\partial w}{\partial t}\left(x^{\prime}, t^{\prime}\right)-\frac{1}{2 \lambda} \int_{\left(x^{\prime}, t-\left|x-x^{\prime}\right|\right) \in S} \frac{\partial w}{\partial t}\left(x^{\prime}, t-\left|x-x^{\prime}\right|\right) d x^{\prime} \\
-\frac{1}{2 \lambda \sqrt{\lambda}} \int_{T_{x, t}} g_{0}^{\prime}\left(\frac{\sqrt{\left(t-t^{\prime}\right)^{2}-\left(x-x^{\prime}\right)^{2}}}{\sqrt{\lambda}}\right)\left(t-t^{\prime}\right)\left(w \cdot 1_{S}\right)(x, t) d x^{\prime} d t .
\end{aligned}
$$

Reasoning as for (165), we can prove under assumption (164) that

$$
\left|\left(\frac{\partial u_{\lambda}}{\partial t}-\frac{\partial u_{0}}{\partial t}\right)(x, t)\right|=o(1)
$$

or

$$
\left|\frac{\partial u_{\lambda}}{\partial t}(x, t)-w_{t}\left(x_{0}, t_{0}\right) \cos \frac{t-t_{0}-m\left(x-x_{0}\right)}{\sqrt{\lambda\left(1-m^{2}\right)}}\right|=o(1),
$$

and, in particular

$$
\begin{aligned}
\lim _{\lambda \rightarrow 0} \frac{\partial u_{\lambda}}{\partial t}\left(x_{0}, t_{0}\right. & \left.+(\pi-\varepsilon) \sqrt{\lambda\left(1-m^{2}\right)}\right)=+w_{t}\left(x_{0}, t_{0}\right) \cos (\pi-\varepsilon) \\
& \text { on }\left\{x_{0}: \tau^{\prime}\left(x_{0}\right)<1 \text { and } w_{t}\left(x_{0}, \tau\left(x_{0}\right) ; Q_{i}\right)<0, i=1, \cdots, 4\right\} .
\end{aligned}
$$


Analogously,

$$
\begin{aligned}
\lim _{\lambda \rightarrow 0} \frac{\partial u_{\lambda}}{\partial x}\left(x_{0}, t_{0}+(\pi-\varepsilon) \sqrt{\lambda\left(1-m^{2}\right)}\right)=w_{x}\left(x_{0}, t_{0}\right) \cos (\pi-\varepsilon) \\
\quad \text { on }\left\{x_{0}: \tau^{\prime}\left(x_{0}\right)<1 \text { and } w_{t}\left(x_{0}, \tau\left(x_{0}\right) ; Q_{i}\right)<0, i=1, \cdots, 4\right\},
\end{aligned}
$$

and (167) and (168) in turn imply:

$$
\begin{aligned}
& \lim _{\lambda \rightarrow 0} \frac{\partial u_{\lambda}}{\partial \xi}\left(x_{0}, t_{0}+(\pi-\varepsilon) \sqrt{\lambda\left(1-m^{2}\right)}\right)=w_{\xi}\left(x_{0}, t_{0}\right) \cos (\pi-\varepsilon), \\
& \lim _{\lambda \rightarrow 0} \frac{\partial u_{\lambda}}{\partial \eta}\left(x_{0}, t_{0}+(\pi-\varepsilon) \sqrt{\lambda\left(1-m^{2}\right)}\right)=w_{\eta}\left(x_{0}, t_{0}\right) \cos (\pi-\varepsilon) .
\end{aligned}
$$

Therefore, the limit $\bar{u}$ of $u$ satisfies:

$$
\frac{\partial \bar{u}}{\partial t}(x, \tau(x))=-\frac{\partial w}{\partial t}(x, \tau(x)) \text { a.e. on }\left\{x:\left|\tau^{\prime}(x)\right|<1\right\} .
$$

This proves that $\bar{u}$ is indeed the solution of $\left(\mathrm{P}_{\infty}\right)$.

6. Conclusion. There are still many open problems which can be conveniently listed at this point. The main one is to prove existence of an energy conserving solution when the obstacle is not assumed to be concave, as was the case in [5].

An obstruction to the proof of existence is that the lines of influence might cluster, and we do not know how to extend the solution after they have clustered.

But there is a more fundamental problem: the whole model relies on the assumption that the motion is transverse: how well is this assumption satisfied when the obstacle is not parallel to the rest position of the string? A better model might be needed; it should be at the same time realistic and tractable.

Another class of problems is the study of the qualitative properties of the system that we consider: periodicity, almost periodicity, for instance; for a first set of results in this direction, see [7].

\section{REFERENCES}

[1] L. AMERIo, Su un problema di vincoli unilaterali per l'equazione non omogenea della corda vibrante, Pubbl. IACD, 190 (1976), pp. 3-11.

[2] A. BAMBERGER, personal communication, 1978.

[3] _ Thèse d'état, Université P. et M. Curie, Paris, 1978.

[4] C. CITRINI, Discontinuous solutions of a nonlinear hyperbolic equation with unilateral constraints, Manuscripta Math., 29 (1979), pp. 323-352.

[5] M. SCHATZMAN, $A$ hyperbolic problem of second order with unilateral constraints: the vibrating string with a concave obstacle, J. Math. Anal. Appl., 73 (1980), pp. 138-191.

[6] __ Un problème hyperbolique du 2ème ordre avec constraintes unilatérales: la corde vibrante avec obstacle ponctuel, J. Differential Equations, 36 (1980), pp. 295-334.

[7] H. Cabannes and A. Haraux, to appear. 